\title{
A SPECTRAL SEQUENCE IN SURGERY THEORY AND MANIFOLDS WITH FILTRATIONS
}

\author{
YU. V. MURANOV, D. REPOVŠ, AND R. JIMENEZ
}

\begin{abstract}
In 1978 Cappell and Shaneson pointed out interesting properties of the Browder-Livesay invariants, which are analogous to the differentials of a certain spectral sequence. Such a spectral sequence was constructed by Hambleton and Kharshiladze in 1991. The main step of the construction of the spectral sequence consists in constructing an infinite filtration of spectra, in which, as is well known, only the first two spectra have a clear geometric meaning. In the present paper a geometric interpretation is given to all the spectra of the filtration in the Hambleton-Kharshiladze construction. Surgery obstruction groups for a system of embedded manifolds are introduced, and it is proved that the spectra realizing these groups coincide with the spectra in the Hambleton-Kharshiladze filtration. The algebraic and geometric properties of these groups and their connections with classical surgery theory are described. An isomorphism between these groups and the Browder-Quinn surgery obstruction groups for stratified manifolds is established. The results obtained are applied to the problem of realization of elements of the Wall groups by normal maps of closed manifolds and to the study of the iterated Browder-Livesay invariants.
\end{abstract}

\section{INTRODUCTION}

Let $X^{n}$ be a closed $n$-dimensional $C A T$-manifold $(C A T=T O P, P L, D I F F)$ with fundamental group $\pi=\pi_{1}(X)$, which is given together with an orientation homomorphism $w: \pi_{1}(X) \rightarrow\{ \pm 1\}$. In what follows we assume that all groups are given with orientation homomorphisms, but we will not indicate this in the notation unless it is necessary.

The main problem of geometric topology is the description of all the closed $n$-dimensional $C A T$-manifolds that are (simply) homotopy equivalent to $X^{n}$. More precisely, let $f: M^{n} \rightarrow X^{n}$ be a simple homotopy equivalence of $C A T$-manifolds. The structure set $\mathcal{S}^{C A T}(X)$ is the set of the equivalence classes of the $s$-cobordant $C A T$-manifolds that are simply homotopy equivalent to $X^{n}$ (see [34, 29] and 30, p. 542]). The elements of $\mathcal{S}^{C A T}(X)$ are called s-triangulations of the manifold $X$.

The Sullivan-Novikov-Wall exact sequence

$$
\cdots \rightarrow L_{n+1}(\pi) \rightarrow \mathcal{S}^{C A T}(X) \rightarrow[X, G / C A T] \stackrel{\sigma}{\rightarrow} L_{n}(\pi) \rightarrow \cdots
$$

is the main tool for describing the structure set $\mathcal{S}^{C A T}(X)$ (see [34, 30]).

2000 Mathematics Subject Classification. Primary 57R67; Secondary 19J25, 57Q10, 57Q15.

Key words and phrases. Spectral sequence, Browder-Livesay invariants, filtration of spectra, surgery obstruction groups, stratified manifold, Wall groups, fundamental group, simple homotopy equivalence, splitting problem, normal fibration, homotopy group.

The first author was supported by the Russian Foundation for Basic Research (grant no. 05-0100993).

The second author was supported by the MESS Research Programme (no. P1-0292-0101-04).

The third author was supported by grants from CONACyT, DGAPA-UNAM, Fulbright-Garcia Robles, and University of Wisconsin-Madison. 
In what follows we shall consider only topological manifolds $(C A T=T O P)$ and the groups $L_{*}(\pi)=L_{*}^{s}(\pi)$ that determine the obstructions to surgery up to a simple homotopy equivalence (see [34, $\S 10]$ and [30]). In order to describe the structure set $\mathcal{S}^{T O P}(X)$ we must calculate the set of normal invariants $[X, G / T O P]$, the surgery obstruction groups $L_{n}(\pi)$, and the map $\sigma$ in (1.1). To describe the map $\sigma$ we need to know which elements of the group $L_{n}(\pi)$ are realized by normal maps of closed manifolds.

The Ranicki algebraic exact sequence of surgery theory

$$
\cdots \rightarrow L_{m+1}\left(\pi_{1}(X)\right) \rightarrow \mathcal{S}_{m+1}(X) \rightarrow H_{m}\left(X ; \mathbf{L}_{\bullet}\right) \stackrel{\sigma}{\rightarrow} L_{m}\left(\pi_{1}(X)\right) \rightarrow \cdots
$$

is defined for any topological space $X$ (see [29, 30]). In particular, this sequence defines the assembly map

$$
H_{n}\left(K(\pi, 1) ; \mathbf{L}_{\bullet}\right) \stackrel{A}{\rightarrow} L_{n}(\pi),
$$

and the image $\operatorname{Im} A \subset L_{n}(\pi)$ is the subgroup consisting of the elements that are realized by normal maps of closed manifolds (see, for example, [34, §13]).

If the space $X$ is simply homotopy equivalent to a topological $n$-dimensional manifold $(n \geq 5)$, then the exact sequence (1.1) is isomorphic to the corresponding part of (1.2). The exact sequence (1.2) is realized at the spectrum level by the map

$$
X_{+} \wedge \mathbf{L}_{\bullet} \rightarrow \mathbb{L}\left(\pi_{1}(X)\right),
$$

where $\mathbb{L}\left(\pi_{1}(X)\right)$ is the $L$-spectrum of surgery theory for the fundamental group $\pi_{1}(X)$ with

$$
\pi_{n}\left(\mathbb{L}\left(\pi_{1}(X)\right)\right) \cong L_{n}\left(\pi_{1}(X)\right),
$$

while $\mathbf{L}_{\bullet}$ is the 1 -connected covering of the $\Omega$-spectrum $\mathbb{L}(\mathbb{Z})$ such that $\mathbf{L}_{\bullet} \simeq G / T O P$.

In particular, for the manifold $X$ we have the isomorphisms

$$
\mathcal{S}_{n+1}(X) \cong \mathcal{S}^{T O P}(X) \text { and } H_{n}\left(X ; \mathbf{L}_{\bullet}\right) \cong[X, G / T O P]
$$

(see 29, 30]).

The methods for calculating the structure set $\mathcal{S}^{T O P}(X)$ are completely different, depending on whether $\pi$ is a finite or infinite group. The case of an infinite group is closely related to Novikov's conjecture (see, for example, [20]). In the case of a finite group with trivial orientation the solution of this question for the special case of decorations (the case of intermediate groups $L^{\prime}$ ) was obtained in [23]. The fundamental results in [23] are based on the analysis of the assembly map and on the methods used in [16, 21]. The methods developed in [9, 15, 16, 21] allow one to prove that, for an arbitrary group $\pi$, certain elements of the Wall groups $L_{n}(\pi)$ that do not belong to the image of the natural map $L_{n}(1) \rightarrow L_{n}(\pi)$ are not realizable.

In particular, in 21] Hambleton solved the corresponding problem for Novikov's projective groups $L_{*}^{p}$. These methods are mainly algebraic and are based on the algebraic theory of splitting homotopy equivalences along submanifolds.

Let $Y \subset X$ be a locally flat submanifold of codimension $q$ in a closed topological manifold $X$ of dimension $n$. A simple homotopy equivalence $f: M \rightarrow X$ splits along the submanifold $Y$ if it is homotopy equivalent to a map $g$ that is transversal to $Y$ with submanifold $N=g^{-1}(Y) \subset M$, and the restrictions

$$
\left.g\right|_{N}: N \rightarrow Y,\left.\quad g\right|_{(M \backslash N)}: M \backslash N \rightarrow X \backslash Y
$$

are simple homotopy equivalences. Let $U$ be a tubular neighbourhood of the submanifold $Y$ in $X$ with boundary $\partial U$. We denote by

$$
F=\left(\begin{array}{ccc}
\pi_{1}(\partial U) & \rightarrow & \pi_{1}(X \backslash Y) \\
\downarrow & & \downarrow \\
\pi_{1}(U) & \rightarrow & \pi_{1}(X)
\end{array}\right)
$$


the push-out square of fundamental groups with orientations. There exists a splitting obstruction group $L S_{n-q}(F)$ (see [34, 30]), which depends only on $n-q \bmod 4$ and the square $F$.

Let $(f, b): M \rightarrow X$ be a normal map with map of bundles $b: \nu_{M} \rightarrow \xi$ covering $f$, where $\xi$ is the topological reduction of the Spivak normal fibration over $X$ [29, 30. In this case the obstruction to the existence of a map $\left(g, b^{\prime}\right)$ with properties (1.5) in the normal bordism class of the map $(f, b)$ is contained in the group $L P_{n-q}(F)$ of surgery obstructions for manifold pairs (see 34, 30] ). This group also depends only on $n-q \bmod 4$ and the square $F$ of fundamental groups.

The basic relation between the $L S_{*^{-}}$and $L P_{*}$-groups and the algebraic exact sequence of surgery theory (1.2) is given by the following commutative diagram [34, $\S 11]$ :

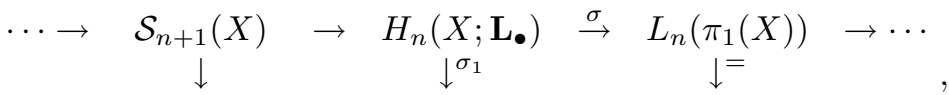

$$
\begin{aligned}
& \cdots \rightarrow L S_{n-q}(F) \quad \rightarrow \quad L P_{n-q}(F) \stackrel{s}{\rightarrow} L_{n}\left(\pi_{1}(X)\right) \stackrel{\partial}{\rightarrow} \cdots
\end{aligned}
$$

where the rows are exact sequences. It follows from (1.7) that the image of the map $\sigma$ is contained in the kernel of the map

$$
\partial: L_{n}\left(\pi_{1}(X)\right) \rightarrow L S_{n-q-1}(F),
$$

which has an explicit geometric description [16, 9].

The lower row in (1.7) is involved in the following braid of exact sequences (see 34, p. 264] and [30, § 7.2]):

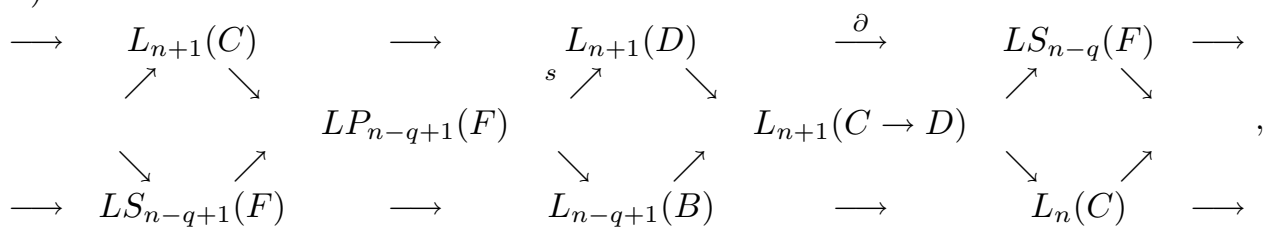

where $A=\pi_{1}(\partial U), B=\pi_{1}(Y), C=\pi_{1}(X \backslash Y)$ and $D=\pi_{1}(X)$.

Now suppose that the manifold pair $(X, Y)$ is a Browder-Livesay pair 4, 9, 13, 16, 21. This means that $Y$ is a one-sided codimension- 1 submanifold of the manifold $X$, and the natural embedding $Y \rightarrow X$ induces an isomorphism of the fundamental groups. In this case the square $F$ of fundamental groups (1.6) has the form

$$
F=\left(\begin{array}{ccc}
\pi_{1}(\partial U) & \rightarrow & \pi_{1}(X \backslash Y) \\
\downarrow & & \downarrow \\
\pi_{1}(Y) & \rightarrow & \pi_{1}(X)
\end{array}\right)=\left(\begin{array}{ccc}
A & \cong & A \\
\downarrow^{i_{-}} & & \downarrow^{i_{+}} \\
B^{-} & \cong & B^{+}
\end{array}\right) .
$$

The orientation of the group $B^{-}$in (1.9) differs from the orientation of the group $B^{+}$ outside the images of the vertical maps (which are embeddings of index 2). All the maps in the square (1.9), except for the lower horizontal map, preserve orientation. The lower isomorphism preserves orientation on the image of $i_{-}$and changes orientation outside this image. In this case we have an isomorphism

$$
L P_{n}(F) \cong L_{n+1}\left(i_{-}^{*}\right),
$$

where $i^{*}: L_{n+1}\left(B^{-}\right) \rightarrow L_{n+1}(A)$ is the transfer map. The group $L S_{*}(F)$ is denoted by $L N_{*}\left(A \rightarrow B^{+}\right)=L N_{*}(A \rightarrow B)$ (see [34]) and is called the Browder-Livesay group.

Cappell and Shaneson [20] proved that, for a Browder-Livesay pair $(X, Y)$, the elements that are not contained in the kernel of the map

$$
\partial: L_{n}\left(\pi_{1}(X)\right) \rightarrow L N_{n-2}\left(\pi_{1}(X \backslash Y) \rightarrow \pi_{1}(X)\right)
$$


cannot be realized by normal maps of closed manifolds.

Diagram (1.8) for a Browder-Livesay pair has an algebraic description (see [21, 31]). This diagram has been studied from algebraic and geometric viewpoints in many papers (see [2, 3, 4, 5, 6, 9, 12, 21, 23, 31).

The spectral sequence in surgery theory was constructed in [12] by using the realization of the commutative diagram (1.8) for a Browder-Livesay pair at the spectrum level. We consider the filtration of spectra in [12],

$$
\cdots \rightarrow \mathbb{X}_{3,0} \rightarrow \mathbb{X}_{2,0} \rightarrow \mathbb{X}_{1,0} \rightarrow \mathbb{X}_{0,0} \rightarrow \mathbb{X}_{-1,0} \rightarrow \cdots
$$

where $\mathbb{X}_{0,0}=\mathbb{L}\left(\pi_{1}(X)\right)$, with $\pi_{n}\left(\mathbb{L}\left(\pi_{1}(X)\right)\right)=L_{n}\left(\pi_{1}(X)\right)$, is the spectrum of surgery theory, and

$$
\mathbb{X}_{1,0}=\Sigma \mathbb{L} P(F)=\mathbb{L}\left(i_{-}^{*}\right)
$$

is the spectrum for the surgery obstruction groups for the manifold pair $(X, Y)$.

The map $s$ in the commutative diagrams (1.7) and (1.8) is induced by the map of spectra $\mathbb{X}_{1,0} \rightarrow \mathbb{X}_{0,0}$ in the filtration (1.10). The other spectra of the filtration are defined inductively by using the construction of the pull-back square, and it was known that they have no geometric meaning. It follows from 12] that the spectral sequence in surgery theory is closely related to the iterated Browder-Livesay invariants and the oozing problem. Other versions of the spectral sequence in surgery theory were obtained in 2 , 17, 18].

Let $Z \subset Y \subset X$ be a triple of closed topological manifolds, so that $X$ has dimension $n, Y$ has codimension $q$ in $X$, and $Z$ has codimension $q^{\prime}$ in $Y$. The surgery obstruction groups $L T_{n-q-q^{\prime}}(X, Y, Z)$ for a manifold triple were introduced in 8 . These groups are realized at the spectrum level by $\mathbb{L} T(X, Y, Z)$. They are a natural generalization of the surgery obstruction groups $L P_{*}$ for manifold pairs. The natural forgetful map $t: L T_{n}(X, Y, Z) \rightarrow L P_{n+1}$ is well defined, which is realized at the spectrum level.

If a triple $(X, Y, Z)$ consists of Browder-Livesay pairs $(X, Y)$ and $(Y, Z)$, then the spectrum $\Sigma^{2} \mathbb{L} T(X, Y, Z)$ coincides with the spectrum $\mathbb{X}_{2,0}$ in the filtration (1.10). The map $\mathbb{X}_{2,0} \rightarrow \mathbb{X}_{1,0}$ of the filtration (1.10) defines the map $t$ at the spectrum level [8].

Now suppose that

$$
X_{k} \subset X_{k-1} \subset \cdots \subset X_{2} \subset X_{1} \subset X_{0}=X
$$

defines a filtration $\mathcal{X}$ of a closed topological manifold $X$ by locally flat embedded submanifolds. We denote by $l_{j}$ the dimension of the submanifold $X_{j}$, and by $q_{j}$ the codimension of $X_{j}$ in $X_{j-1}$ for $1 \leq j \leq k$. We assume that each pair of manifolds in (1.11) is a topological manifold pair in the sense of Ranicki [30, $\S 7.2]$ and that $l_{k} \geq 5$.

For each non-empty subset $B \subset\{k, k-1, \ldots, 2,1,0\}$ the filtration (1.11) defines a subfiltration $\mathcal{X}_{B}$ that is obtained by "forgetting" the submanifolds $X_{j}$ in the filtration (1.10) for $j \in\{k, k-1, \ldots, 2,1,0\} \backslash B$. In particular, for each $0 \leq j \leq k$ the bounded filtration

$$
X_{j} \subset X_{j-1} \subset \cdots \subset X_{2} \subset X_{1} \subset X_{0}=X
$$

is defined, where $B=\{j, j-1, \ldots, 2,1,0\}$. We denote the bounded filtration (1.12) by $\mathcal{X}_{j}$.

In $\S 2$ we define the notion of $s$-triangulation for the filtration $\mathcal{X}$ in (1.11) and prove several technical results. The notion of $s$-triangulation of the filtration $\mathcal{X}$ naturally generalizes the notion of $s$-triangulation of a manifold and of a manifold pair in 30. In particular, we prove that for a manifold triple $Z \subset Y \subset X$ the surgery obstruction groups $L T_{*}(X, Y, Z)$ in [8] coincide with the Browder-Quinn groups $L_{*}^{B Q}$ of the stratified manifold $Z \subset Y \subset X$ (see [14, 35]). 
In $\S 3$, we introduce the groups of obstructions to surgery of a normal map to an $s$-triangulation of the filtration $\mathcal{X}$ in (1.11) and study their properties. We introduce the obstruction groups $L M_{i}^{j}(\mathcal{X})(0 \leq j \leq k)$, which have period 4 with respect to the lower index $i$ and are realized by the spectrum $\mathbb{L} M^{j}(\mathcal{X})$ with $\pi_{i}\left(\mathbb{L} M^{j}(\mathcal{X})\right)=L M_{i}^{j}(\mathcal{X})$. The groups $L M_{*}^{k}(\mathcal{X})$ coincide with the Browder-Quinn stratified $L$-groups $L_{*}^{B Q}(\mathcal{X})$ (see [14, 35]) up to a shift of dimension $*$. The spectrum $\mathbb{L} M^{0}$ coincides with the spectrum $\mathbb{L}\left(\pi_{1}(X)\right)$, the spectrum $\mathbb{L} M^{1}$ coincides with the spectrum $\mathbb{L} P(F)$ for the pair $\left(X_{0}, X_{1}\right)$ (see [30, 34]), and the spectrum $\mathbb{L} M^{2}$ coincides with the spectrum $\mathbb{L} T$ for the triple $\left(X_{0}, X_{1}, X_{2}\right)$ (see [8, 27, 28]).

Let $(f, b):(M \rightarrow X)$ be a normal map into a manifold $X$ with filtration (1.11). For the above-mentioned groups and for $0 \leq j \leq k$, the obstruction $\Theta_{j}(f) \in L M_{l_{j}}^{j}$ is defined. In Theorem 3.9 we prove that this obstruction is trivial if and only if the map $(f, b)$ is normally bordant to an $s$-triangulation of the bounded filtration $\mathcal{X}_{j}$ in (1.12).

Further in $\S 3$, we define the natural neglecting maps

$$
L M_{l_{k}}^{k} \rightarrow L M_{l_{k-1}}^{k-1} \rightarrow \cdots \rightarrow L M_{l_{1}}^{1} \rightarrow L M_{l_{0}}^{0}
$$

which are realized at the spectrum level by the maps

$$
\Sigma^{n-l_{k}} \mathbb{L} M^{k} \rightarrow \Sigma^{n-l_{k-1}} \mathbb{L} M^{k-1} \rightarrow \cdots \rightarrow \Sigma^{n-l_{1}} \mathbb{L} M^{1} \rightarrow \mathbb{L} M^{0} .
$$

In 30] Ranicki introduced the set $\mathcal{S}_{n+1}(X, Y, \xi)$ of homotopy triangulations of a manifold pair $(X, Y)$, where $\xi$ denoted a normal fibration of $Y$ in $X$. This set consists of the concordance classes of the maps $f:(M, N) \rightarrow(X, Y)$ that are split along $Y$. This structure set is a natural generalization of the structure set $\mathcal{S}_{n+1}(X)$ in the exact sequence (1.2) and occurs in the exact sequence

$$
\cdots \rightarrow \mathcal{S}_{n+1}(X, Y, \xi) \rightarrow H_{n}\left(X, \mathbf{L}_{\bullet}\right) \rightarrow L P_{n-q}(F) \rightarrow \cdots
$$

(see [30, $\S 7.2])$. Note that (1.15) is a natural generalization of (1.2) to the case of manifold pairs.

Also in $\S 3$, we introduce structure sets for the filtration (1.11) that generalize the structure sets $\mathcal{S}_{n+1}(X, Y, \xi)$ and $\mathcal{S}_{n+1}(X)$, and we study their properties. Some results for the case of a manifold triple were obtained in $[8,27,28$.

Suppose that in (1.11) all the pairs $X_{i+1} \subset X_{i}$ for $0 \leq i \leq k-1$ are Browder-Livesay pairs. In $\S 4$ we apply our results to study the iterated Browder-Livesay invariants. We describe the connection between the groups we have introduced and the spectral sequence of surgery theory. In Theorem 4.1 we prove that in the case under consideration the filtration (1.14) coincides with the left-hand side of the filtration (1.10) of the Hambleton-

Kharshiladze spectral sequence, starting from $\mathbb{X}_{0,0}$. In addition, in $\S 4$ we describe the relation of the groups $L M_{*}^{i}$ to the problem of realization of elements of the Wall groups by normal maps of closed manifolds.

\section{TECHNiCAL RESUlts}

In this section we present some preliminary results concerning surgery on topological manifolds and the use of $L$-spectra (see 1 , 18, 19, 22, 29, 30, 33]). We give the requisite definitions and prove several technical results.

We consider the case of topological manifolds and follow the notation in [30, § 7.2]. Let $(X, Y, \xi)$ be a manifold pair of codimension $q$ in the sense of Ranicki (see [30, $\S 7.2]$ ). This means that a locally flat submanifold $Y \subset X$ is given together with a normal fibration

$$
\xi=\xi_{Y \subset X}: Y \rightarrow \widetilde{\operatorname{BTOP}}(q)
$$


with the associated $\left(D^{q}, S^{q-1}\right)$ fibration

$$
\left(D^{q}, S^{q-1}\right) \rightarrow(E(\xi), S(\xi)) \rightarrow Y,
$$

and the decomposition of the closed manifold is defined:

$$
X=E(\xi) \cup_{S(\xi)} \overline{X \backslash E(\xi)} .
$$

A topological normal map [30, $\S 7.2]$

$$
((f, b),(g, c)):(M, N) \rightarrow(X, Y)
$$

into the manifold pair $(X, Y, \xi)$ is represented by a normal map $(f, b)$ into the manifold $X$ which is transversal to $Y$ with $N=f^{-1}(Y)$. Here the pair $(M, N)$ is a topological manifold pair with normal fibration

$$
\nu: N \stackrel{\left.f\right|_{N}}{\rightarrow} Y \stackrel{\xi}{\rightarrow} \widetilde{\operatorname{BTOP}}(q) .
$$

In addition, the following conditions hold:

(i) the restriction

$$
\left.(f, b)\right|_{N}=(g, c): N \rightarrow Y
$$

is a normal map;

(ii) the restriction

$$
\left.(f, b)\right|_{P}=(h, d):(P, S(\nu)) \rightarrow(Z, S(\xi))
$$

is a normal map into the pair $(Z, S(\xi))$, where

$$
P=\overline{M \backslash E(\nu)}, \quad Z=\overline{X \backslash E(\xi)} ;
$$

(iii) the restriction

$$
\left.(h, d)\right|_{S(\nu)}: S(\nu) \rightarrow S(\xi)
$$

coincides with the induced map

$$
(g, c)^{!}: S(\nu) \rightarrow S(\xi)
$$

and $(f, b)=(g, c)^{!} \cup(h, d)$.

Normal maps into $(X, Y, \xi)$ are called $t$-triangulations of the manifold pair $(X, Y)$ and the set of the concordance classes of $t$-triangulations of the pair $(X, Y, \xi)$ coincides with the set of $t$-triangulations of the manifold $X$ [30, Proposition 7.2].

By definition, an $s$-triangulation of a manifold pair $(X, Y, \xi)$ in the topological category [30. p. 571] consists of a $t$-triangulation of this pair such that the maps

$$
f: M \rightarrow X, \quad g: N \rightarrow Y, \quad \text { and } \quad(P, S(\nu)) \rightarrow(Z, S(\xi))
$$

are simple homotopy equivalences.

A simple homotopy equivalence $f: M \rightarrow X$ splits along the submanifold $Y$ if $f$ is homotopy equivalent to a map $g$ that is an $s$-triangulation of $(X, Y, \xi)$, that is, satisfies conditions (2.2). In this case, $f$ represents an element of $\mathcal{S}_{n+1}(X, Y, \xi)$. It follows from the definition of an $s$-triangulation of the pair $(X, Y, \xi)$ that the neglecting maps

$$
\begin{array}{ll}
\mathcal{S}_{n+1}(X, Y, \xi) \rightarrow \mathcal{S}_{n+1}(X), & (f, g) \rightarrow f, \\
\mathcal{S}_{n+1}(X, Y, \xi) \rightarrow \mathcal{S}_{n-q+1}(Y), & (f, g) \rightarrow g
\end{array}
$$

are well defined. In the general case the map $\mathcal{S}_{n+1}(X, Y, \xi) \rightarrow \mathcal{S}_{n+1}(X)$ is neither an epimorphism nor a monomorphism [30, p. 571].

We consider a triple of closed topological manifolds $Z^{n-q-q^{\prime}} \subset Y^{n-q} \subset X^{n}$. We assume that each submanifold is locally flat in the ambient manifold and that the submanifolds are equipped with the structure of a normal topological fibration (see 30, pp. 562-563] and [8]). Each manifold pair defines a topological normal fibration, which 
we denote as follows:

$\xi$ for the submanifold $Y$ in $X$,

$\eta$ for the submanifold $Z$ in $Y$, and

$\nu$ for the submanifold $Z$ in $X$.

We denote the spaces with boundary of the associated fibrations (2.1) by $(E(\xi), S(\xi))$, $(E(\eta), S(\eta))$, and $(E(\nu), S(\nu))$, respectively. Let $\left.\xi\right|_{E(\eta)}$ be the restriction of the fibration $\xi$ to the space $E(\eta)$ of the normal fibration $\eta$ with the restriction of the associated fibration (2.1)

$$
\left(D^{q}, S^{q-1}\right) \rightarrow\left(E^{\prime}(\xi), S^{\prime}(\xi)\right) \rightarrow E(\eta) .
$$

Also let $\left.\xi\right|_{S(\eta)}$ be the restriction of the fibration $\xi$ with the restriction of the associated fibration

$$
\left(D^{q}, S^{q-1}\right) \rightarrow\left(E^{\prime \prime}(\xi), S^{\prime \prime}(\xi)\right) \rightarrow S(\eta) .
$$

We assume that the space $E(\nu)$ of the normal fibration $\nu$ is identified with the space $E^{\prime}(\xi)$ of the restriction $\left.\xi\right|_{E(\eta)}$ so that the following conditions on the boundary hold:

$$
S(\nu)=E^{\prime \prime}(\xi) \cup S^{\prime}(\xi) .
$$

Remark 2.1. The existence of normal fibrations for the submanifolds of the manifold triple

$$
Z^{n-q-q^{\prime}} \subset Y^{n-q} \subset X^{n}
$$

that have associated fibrations satisfying conditions (2.3) implies that the triple $Z \subset$ $Y \subset X$ is a $\mathcal{C}$-stratified set in the sense of Browder and Quinn [14.

We denote by $\mathcal{X}$ the filtration of the closed manifold $X^{n}$ by the system of submanifolds (1.11). All the pairs of submanifolds are given together with normal fibrations and the corresponding associated $\left(D^{*}, S^{*-1}\right)$ fibrations (2.1). We assume that for each manifold triple $X_{j} \subset X_{l} \subset X_{m}$ with $k \geq j>l>m \geq 0$ the normal fibrations satisfy requirements analogous to (2.3) for the triple $Z \subset Y \subset X$.

Remark 2.2. Under these assumptions the filtration $\mathcal{X}$ in (1.11) is a $\mathcal{C}$-stratified set in the sense of Browder and Quinn [14 - this follows from Remark 2.1 and 14, Definition 4.2].

A manifold pair $(Y, \partial Y) \subset(X, \partial X)$ of codimension $q$ with boundary was defined in 30 , p. 585]. We have a normal fibration $(\xi, \partial \xi)$ over the pair $(Y, \partial Y)$ and the decomposition

$$
(X, \partial X)=\left(E(\xi) \cup_{S(\xi)} Z, E(\partial \xi) \cup_{S(\partial \xi)} \partial_{+} Z\right),
$$

where $\left(Z ; \partial_{+} Z, S(\xi) ; S(\partial \xi)\right)$ is a manifold triad. Note that here

$$
\partial_{+} Z=\overline{\partial X \backslash E(\partial \xi)}
$$

A topological normal map of a manifold pair with boundary

$$
(f, \partial f):(M, \partial M) \rightarrow(X, \partial X)
$$

defines a normal fibration $(\nu, \partial \nu)$ over the pair $(N, \partial N)$ (see [30, p. 570]), where

$$
(N, \partial N)=\left(f^{-1}(Y),(\partial f)^{-1}(\partial Y)\right) .
$$

We have the decomposition

$$
(M, \partial M)=\left(E(\nu) \cup_{S(\nu)} P, E(\partial \nu) \cup_{S(\partial \nu)} \partial_{+} P\right),
$$

where $\left(P ; \partial_{+} P, S(\nu) ; S(\partial \nu)\right)$ is a manifold triad.

We now define the filtration $(\mathcal{X}, \partial \mathcal{X})$ for the case of manifolds with boundary to be the filtration

$$
\left(X_{k}, \partial X_{k}\right) \subset\left(X_{k-1}, \partial X_{k-1}\right) \subset \cdots \subset\left(X_{0}, \partial X_{0}\right)=(X, \partial X),
$$


where all the manifold pairs with boundary satisfy conditions analogous to (2.4). We also assume that the normal fibrations of the manifolds of the filtration and of the boundaries satisfy conditions analogous to (2.3).

Remark 2.3. Under the assumptions made above, the filtration $\mathcal{X}$ in (1.11) gives rise to the filtration of manifolds with boundary

$$
\left(\overline{X_{k-1} \backslash X_{k}}, \partial\left(\overline{X_{k-1} \backslash X_{k}}\right)\right) \subset \cdots \subset\left(\overline{X \backslash X_{k}}, \partial\left(\overline{X \backslash X_{k}}\right)\right) .
$$

This filtration is a $\mathcal{C}$-stratified manifold with boundary in the sense of [14, 35. We denote this filtration by $\overline{\mathcal{X}_{k}}=\overline{\mathcal{X}}$. We can similarly construct the filtration $\overline{\mathcal{X}_{j}}$ using the bounded filtration (1.12).

Definition. A topological normal map $(f, b): M \rightarrow X$ that is topologically transversal to each submanifold of the filtration with transversal inverse images $M_{0}=M$ and $M_{i}=$ $f^{-1}\left(X_{i}\right)$ for $0 \leq i \leq k$ is called a topological normal map into the filtration $\mathcal{X}$ in (1.11) (a t-triangulation of the filtration $\mathcal{X}$ ). In addition we assume that the restriction to each pair of submanifolds $\left(M_{j}, M_{l}\right)(j \geq l)$ is a topological normal map into the manifold pair $\left(X_{j}, X_{l}\right)$. A bordism between such maps is naturally defined and the equivalence classes are denoted by $\mathcal{T}(\mathcal{X})$ (see [14, 35]).

It is clear that a $t$-triangulation of the filtration $\mathcal{X}$ defines a $t$-triangulation of the bounded filtration $\mathcal{X}_{B}$ for any non-empty subset $B \subset\{k, k-1, \ldots, 2,1,0\}$. In particular, for each submanifold $X_{j}$ in this filtration we have the neglecting map from $\mathcal{T}(\mathcal{X})$ into the set $\left[X_{j}, G / T O P\right]$ of normal maps into the manifold $X_{j}$.

Proposition 2.4 ([14, 30]). The natural neglecting map

$$
\mathcal{T}(\mathcal{X}) \rightarrow[X, G / T O P]
$$

is an isomorphism.

Proof. Use the topological transversality (see [14, 30, Proposition 7.2.3], and [35]) and induction on the number of elements of the filtration.

Definition. A $t$-triangulation $(f, b): M \rightarrow X$ of the filtration $\mathcal{X}$ in (1.11) is an s-triangulation of the filtration $\mathcal{X}$ if the constituent normal maps of the pairs

$$
\left(M_{j}, M_{l}\right) \rightarrow\left(X_{j}, X_{l}\right), \quad 0 \leq j<l \leq k,
$$

are $s$-triangulations, that is, they satisfy properties that are analogous to $(2.2)$ for the manifold pair $(X, Y)$.

Proposition 2.5. Suppose that a t-triangulation $(f, b): \mathcal{M} \rightarrow \mathcal{X}$ defines an s-triangulation $f_{k}: \mathcal{M}_{k} \rightarrow \mathcal{X}_{k}$, where the filtration $\mathcal{X}_{k}$ is obtained from $\mathcal{X}$ by forgetting the submanifold $X_{k}$, and similarly for $\mathcal{M}_{k}$. Suppose that the restriction $\left.f\right|_{M_{k}}$ is an s-triangulation of the pair $\left(X_{k-1}, X_{k}\right)$. Then $(f, b)$ is an s-triangulation of the filtration $\mathcal{X}$.

Proof. It is sufficient to prove that for each submanifold $X_{j} \subset X, 0 \leq j \leq k-2$, the restriction map

$$
\left.f\right|_{M_{j} \backslash M_{k}}:\left(M_{j} \backslash M_{k}\right) \rightarrow X_{j} \backslash X_{k}
$$

is a simple homotopy equivalence. But the conditions on the boundaries of tubular neighbourhoods given in (2.3) are satisfied for the triple $X_{k} \subset X_{k-1} \subset X_{j}$. For such a triple the result was proved in [27, Proposition 2.1], using the properties of the simple homotopy equivalence of triads given in 19 . 
The groups $L T_{*}(X, Y, Z)$ and the map

$$
\Theta_{*}(f, b):[X, G / T O P] \rightarrow L T_{n-q-q^{\prime}}(X, Y, Z)
$$

were defined in [8] so that a normal map $(f, b)$ is normally bordant to an $s$-triangulation of the triple $(X, Y, Z)$ if and only if $\Theta_{*}(f, b)=0$ (for $\left.n-q-q^{\prime} \geq 5\right)$.

These groups were defined at the spectrum level. First we recall the requisite facts about applications of spectra in $L$-theory.

A spectrum $\mathbb{E}$ is given by a set of $C W$-complexes $\left\{\left(E_{n}, *\right)\right\}, n \in \mathbb{Z}$, with a set of cell maps $\left\{\epsilon_{n}: S E_{n} \rightarrow E_{n+1}\right\}$, where $S E_{n}$ is the suspension of the space $E_{n}$ 33. The conjugate maps $\epsilon_{n}^{\prime}: E_{n} \rightarrow \Omega E_{n+1}$ are defined (see 33) and the spectrum $\mathbb{E}$ is an $\Omega$ spectrum if all the conjugate maps are homotopy equivalences. Let $\Sigma \mathbb{E}$ be the spectrum with $\{\Sigma \mathbb{E}\}_{n}=E_{n+1}$ and $\{\Sigma \epsilon\}_{n}=\epsilon_{n+1}$. The functor $\Sigma$ has the inverse functor $\Sigma^{-1}$, and the iterated functors $\Sigma^{k}, k \in \mathbb{Z}$, are defined on the category of spectra. For any spectrum $\mathbb{E}$ we have an isomorphism

$$
\pi_{n}(\mathbb{E})=\pi_{n+k}\left(\Sigma^{k} \mathbb{E}\right)
$$

of homotopy groups. We now recall that in the homotopy theory of spectra there is an equivalence between pull-back and push-out squares. A homotopy commutative square of spectra

$$
\begin{array}{lll}
\mathbb{G} & \rightarrow & \mathbb{H} \\
\downarrow & & \downarrow \\
\mathbb{E} & \rightarrow & \mathbb{F}
\end{array}
$$

is a pull-back square if the fibres of the horizontal (vertical) maps are naturally homotopy equivalent 33. The square (2.7) is a push-out square if the cofibres of the horizontal (vertical) maps are naturally homotopy equivalent.

Natural maps of $L$-groups such as transfer and inducing maps are realized at the spectrum level. A homomorphism of oriented groups $f: \pi \rightarrow \pi^{\prime}$ induces a cofibration of $\Omega$-spectra (see [22])

$$
\mathbb{L}(\pi) \rightarrow \mathbb{L}\left(\pi^{\prime}\right) \rightarrow \mathbb{L}(f),
$$

where $\pi_{n}(\mathbb{L}(\pi))=L_{n}(\pi)$, and similarly for other spectra. The homotopy long exact sequence of the cofibration (2.8) gives rise to the relative exact sequence of $L$-groups

$$
\cdots \rightarrow L_{n}(\pi) \rightarrow L_{n}\left(\pi^{\prime}\right) \rightarrow L_{n}(f) \rightarrow L_{n-1}(\pi) \rightarrow \cdots .
$$

For a fibration $p: E^{m+n} \rightarrow X^{n}$ over a closed topological manifold $X^{n}$, the transfer map

$$
p^{*}: L_{n}\left(\pi_{1}(X)\right) \rightarrow L_{n+m}\left(\pi_{1}(E)\right)
$$

is defined (see $25,26,34,35]$ ), which is realized at the spectrum level by the map of $\Omega$-spectra

$$
p^{!}: \mathbb{L}\left(\pi_{1}(X)\right) \rightarrow \Sigma^{-m} \mathbb{L}\left(\pi_{1}(E)\right) .
$$

For a manifold pair $(X, Y)$ we have the homotopy commutative diagram of spectra

$$
\begin{array}{cccc}
\mathbb{L}\left(\pi_{1}(Y)\right) \underset{p^{!}}{\stackrel{p_{1}^{!}}{\longrightarrow}} & \Sigma^{-q} \mathbb{L}\left(\pi_{1}(\partial U) \rightarrow \pi_{1}(U)\right) & \stackrel{\alpha}{\longrightarrow} & \Sigma^{-q} \mathbb{L}\left(\pi_{1}(X \backslash Y) \longrightarrow \pi_{1}(X)\right) \\
& \Sigma^{1-q} \mathbb{L}\left(\pi_{1}(\partial U)\right) & \stackrel{\beta}{\longrightarrow} & \Sigma^{1-q} \mathbb{L}\left(\pi_{1}(X \backslash Y)\right)
\end{array}
$$

in which the maps $p^{!}$and $p_{1}^{!}$are transfer maps and the right-hand horizontal maps are induced by the horizontal maps in the square $F$ in (1.6). The two vertical maps in (2.10) 
are obtained from the extended cofibred sequences (2.8) for the vertical maps in the square $F$ in (1.6). The spectrum $\mathbb{L} S(F)$ is the homotopy cofibre of the map

$$
\Sigma^{-1}\left(\alpha p_{1}^{!}\right): \Sigma \mathbb{L}\left(\pi_{1}(Y)\right) \rightarrow \Sigma^{-q-1} \mathbb{L}\left(\pi_{1}(X \backslash Y) \rightarrow \pi_{1}(X)\right),
$$

and the spectrum $\mathbb{L} P(F)$ is the homotopy cofibre of the map

$$
\Sigma^{-1}\left(\beta p^{!}\right): \Sigma^{-1} \mathbb{L}\left(\pi_{1}(Y)\right) \rightarrow \Sigma^{-q} \mathbb{L}\left(\pi_{1}(X \backslash Y)\right) .
$$

We have the isomorphisms (see [1, 3, 6, 8])

$$
\pi_{n}(\mathbb{L} S(F)) \cong L S_{n}(F), \quad \pi_{n}(\mathbb{L} P(F)) \cong L P_{n}(F) .
$$

We denote the set of the concordance classes of $s$-triangulations of the manifold pair $(X, Y, \xi)$ by $\mathcal{S}_{n+1}(X, Y, \xi)$ (see [30]).

For a triple of closed topological manifolds $Z \subset Y \subset X$, we consider the square of fundamental groups with orientation in the splitting problem for the manifold pair $Z \subset Y:$

$$
\Psi=\left(\begin{array}{ccc}
\pi_{1}(\partial V) & \rightarrow & \pi_{1}(Y \backslash Z) \\
\downarrow & & \downarrow \\
\pi_{1}(Z) & \rightarrow & \pi_{1}(Y)
\end{array}\right)
$$

We consider the commutative diagram (see [30, 34])

$$
\begin{aligned}
& \cdots \rightarrow \mathcal{S}_{n+1}(X, Y, \xi) \quad \rightarrow \quad H_{n}\left(X ; \mathbf{L}_{\bullet}\right) \quad \stackrel{\sigma_{1}}{\rightarrow} L P_{n-q}(F) \quad \rightarrow \cdots
\end{aligned}
$$

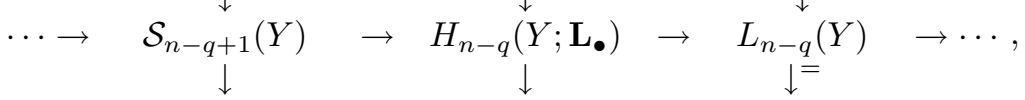

$$
\begin{aligned}
& \cdots \rightarrow \quad L S_{k}(\Psi) \quad \rightarrow \quad L P_{k}(\Psi) \quad \rightarrow \quad L_{n-q}(Y) \quad \rightarrow \cdots
\end{aligned}
$$

in which $k=n-q-q^{\prime}$ is the dimension of $Z$ and the rows and columns are exact sequences. Note that the two lower rows represent (1.7) for the manifold pair $(Y, Z)$. Diagram (2.15) is realized at the spectrum level (see [1, 8]).

In particular, the composite

$$
L P_{n-q+1}(F) \rightarrow \mathcal{S}_{n+1}(X, Y, \xi) \rightarrow \mathcal{S}_{n-q+1}(Y) \rightarrow L S_{n-q-q^{\prime}}(\Psi)
$$

of the maps in diagram (2.15) is realized by the composite $\mathbf{v}$ of the maps of spectra

$$
\mathbb{L} P(F) \rightarrow \Sigma^{-q} \mathbb{S}(X, Y, \xi) \rightarrow \mathbb{S}(Y) \rightarrow \Sigma^{q^{\prime}+1} \mathbb{L} S(\Psi),
$$

where

$$
\pi_{n}(\mathbb{S}(X, Y, \xi))=\mathcal{S}_{n}(X, Y, \xi), \quad \pi_{n}(\mathbb{S}(Y))=\mathcal{S}_{n}(Y)
$$

The spectrum $\mathbb{L} T(X, Y, Z)$ is the homotopy cofibre of the map

$$
\Sigma^{-q^{\prime}-1} \mathbf{v}: \Sigma^{-q^{\prime}-1} \mathbb{L} P(F) \rightarrow \mathbb{L} S(\Psi),
$$

and by definition $L_{n}(X, Y, Z)=\pi_{n}(\mathbb{L} T(X, Y, Z)$ ) (see [8]). The homotopy long exact sequence of the cofibration (2.16) gives rise to the exact sequence

$$
\cdots \rightarrow L P_{n-q+1}(F) \rightarrow L S_{n-q-q^{\prime}}(\Psi) \rightarrow L T_{n-q-q^{\prime}}(X, Y, Z) \rightarrow \cdots .
$$

The manifold triple $Z \subset Y \subset X$ is a stratified topological manifold (see [14, 35]), which we denote by $\mathcal{X}$. Consequently, the Browder-Quinn stratified $L$-groups $L^{B Q}(\mathcal{X})$ are defined. These groups are realized at the spectrum level, and we will use our notation to recall their inductive definition, which was given in [35, p. 129]. According to Remark 2.3 the triple $Z \subset Y \subset X$ defines the pair of manifolds with boundary

$$
(Y \backslash Z, \partial(Y \backslash Z)) \subset(X \backslash Z, \partial(X \backslash Z)),
$$

where $\partial(Y \backslash Z) \subset \partial(X \backslash Z)$ is the manifold pair that coincides with the natural decomposition of the boundary of a tubular neighbourhood of $Z$ in $X$. We denote by $F_{Z}$ the 
square of fundamental groups in the splitting problem with respect to the boundary for the manifold pair (2.18), and by $F_{U}$ the analogous square for the pair of closed manifolds

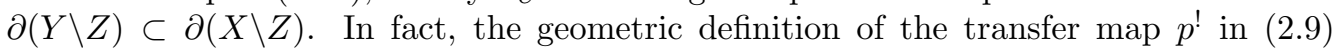
and (2.10) for the pair $Z \subset X$ (see [25, 26, 30, 34]) gives the map

$$
p^{\#}: L_{n-q-q^{\prime}}\left(\pi_{1}(Z)\right) \rightarrow L P_{n-q-1}\left(F_{U}\right)
$$

which is realized at the spectrum level (see [35]) by the map

$$
\mathbf{p}^{\#}: \mathbb{L}\left(\pi_{1}(Z)\right) \rightarrow \Sigma^{-q^{\prime}+1} \mathbb{L} P\left(F_{U}\right) .
$$

We consider the composite of the map $\mathbf{p}^{\#}$ in (2.20) with the map of spectra

$$
\text { b : } \Sigma^{-q^{\prime}+1} \mathbb{L} P\left(F_{U}\right) \rightarrow \Sigma^{-q^{\prime}+1} \mathbb{L} P\left(F_{Z}\right)
$$

that is induced by the embedding of the boundary in (2.18). We obtain the cofibration of spectra 35

$$
\mathbf{p}^{\#} \mathbf{b}: \mathbb{L}\left(\pi_{1}(Z)\right) \rightarrow \Sigma^{-q^{\prime}+1} \mathbb{L} P\left(F_{Z}\right) \rightarrow \Sigma^{-q-q^{\prime}+1} \mathbb{L}^{B Q}(\mathcal{X})
$$

with the cofibre $\Sigma^{-q-q^{\prime}+1} \mathbb{L}^{B Q}(\mathcal{X})$. By definition (see [14, 35]),

$$
\pi_{n}\left(\mathbb{L}^{B Q}(\mathcal{X})\right)=L_{n}^{B Q}(\mathcal{X})
$$

For the groups $L_{n}^{B Q}$ the index $n$ is equal to the dimension taken mod 4 of the larger manifold of the filtration (see [14, 35]). In the case of the surgery obstruction groups $L P_{n}$, Wall and Ranicki (see [30, 34]) used the index $n$ that corresponds to the dimension of the smaller manifold in the pair. Similarly to Wall and Ranicki, for the surgery obstruction groups $L T_{n}$ for a manifold triple, the index $n$ is equal to the dimension of the lower manifold of the filtration.

Remark 2.6. For the manifold triple $(X, Y, Z)$ the homotopy long exact sequence of the cofibration (2.21) gives rise to the exact sequence of obstruction groups

$$
\cdots \rightarrow L_{n-q-q^{\prime}}\left(\pi_{1}(Z)\right) \rightarrow L P_{n-q-1}\left(F_{Z}\right) \rightarrow L_{n-1}^{B Q}(\mathcal{X}) \rightarrow \cdots
$$

where $\mathcal{X}$ denotes the filtration $Z \subset Y \subset X$.

We denote by $\Phi$ the square of fundamental groups in the splitting problem for the pair $(X, Z)$. The groups $L P_{*}(\Phi)$ occur in the exact sequence (see [30, 34])

$$
\cdots \rightarrow L_{n}\left(\pi_{1}(X \backslash Y)\right) \rightarrow L P_{n-q-q^{\prime}}(\Phi) \rightarrow L_{n-q-q^{\prime}}\left(\pi_{1}(Z)\right) \rightarrow \cdots,
$$

which is realized at the spectrum level, similarly to (2.12), by the cofibration of spectra

$$
\mathbb{L} P(\Phi) \rightarrow \mathbb{L}\left(\pi_{1}(Z)\right) \rightarrow \Sigma^{-q-q^{\prime}+1} \mathbb{L}\left(\pi_{1}(X \backslash Z)\right) .
$$

By [8, Theorem 2] the groups $L T_{*}$ occur in the commutative diagram of exact sequences

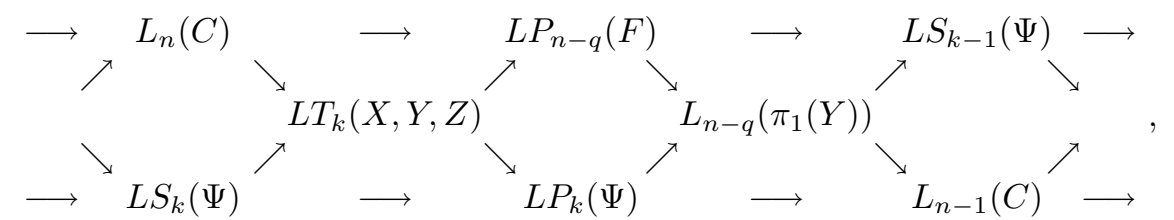

where $k=n-q-q^{\prime}$ and $C=\pi_{1}(X \backslash Y)$. The diagram (2.25) is realized at the spectrum level and contains the exact sequence

$$
\cdots \rightarrow L_{n}\left(\pi_{1}(X \backslash Y)\right) \rightarrow L T_{n-q-q^{\prime}}(X, Y, Z) \rightarrow L P_{n-q-q^{\prime}}(\Psi) \rightarrow \cdots .
$$

The exact sequence $(2.26)$ is realized at the spectrum level by the cofibration

$$
\mathbb{L} T(X, Y, Z) \rightarrow \mathbb{L} P(\Psi) \rightarrow \Sigma^{-q-q^{\prime}+1} \mathbb{L}\left(\pi_{1}(X \backslash Z)\right) .
$$


Proposition 2.7. We have the commutative diagram (2.28)

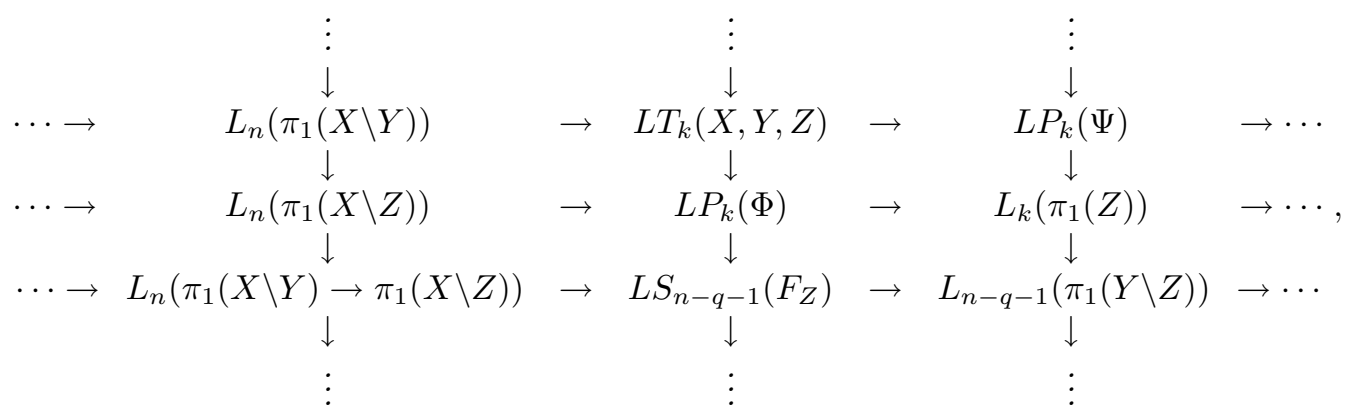

where $k=n-q-q^{\prime}$. The diagram (2.28) is realized at the spectrum level. All the maps in the square

$$
\begin{array}{ccc}
L T_{*}(X, Y, Z) & \rightarrow & L P_{*}(\Psi) \\
\downarrow & & \downarrow \\
L P_{*}(\Phi) & \rightarrow & L_{*}\left(\pi_{1}(Z)\right)
\end{array}
$$

of the diagram (2.28) are natural forgetful maps. The two top horizontal rows in (2.28) coincide with the exact sequences (2.26) and (2.23).

Proof. Forgetting the submanifold $Y$ induces the natural maps

$$
L T_{*}(X, Y, Z) \rightarrow L P_{*}(\Phi) \text { and } L P_{*}(\Psi) \rightarrow L_{*}\left(\pi_{1}(Z)\right),
$$

which are induced by the map of spectra in (2.25) and (2.27). Similarly to (2.25), the forgetful map $L P_{*}(\Psi) \rightarrow L_{*}\left(\pi_{1}(Z)\right)$ is realized at the spectrum level. The forgetful map $L T_{*}(X, Y, Z) \rightarrow L P_{*}(\Phi)$ is realized at the spectrum level according to [27, Theorem 3.5]. This map is involved in the exact sequence

$$
\cdots \rightarrow L T_{n-q-q^{\prime}}(X, Y, Z) \rightarrow L P_{n-q-q^{\prime}}(\Phi) \rightarrow L S_{n-q-1}\left(F_{Z}\right) \rightarrow \cdots .
$$

Hence we have the homotopy commutative diagram of spectra

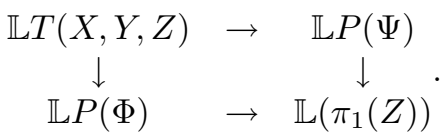

We consider the infinite homotopy commutative diagram of spectra

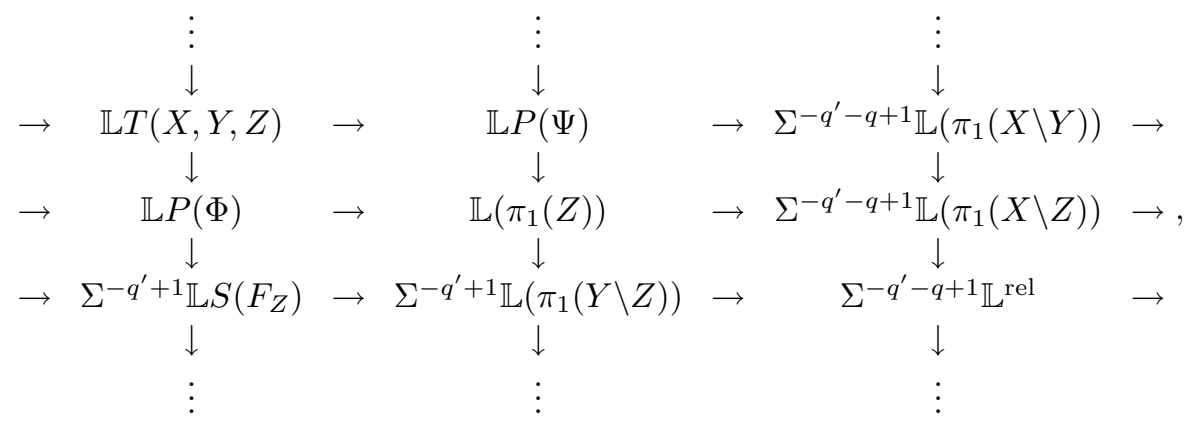

where $\mathbb{L}^{\text {rel }}=\mathbb{L}\left(\pi_{1}(X \backslash Y) \rightarrow \pi_{1}(X \backslash Z)\right)$. This diagram is obtained from the homotopy commutative diagram (2.31) by considering the cofibrations defined by all the maps in $(2.31)$ (see [3, 33]). An application of $\pi_{0}$ to (2.32) produces the commutative diagram (2.28). 
We now recall the following technical result in 3 .

Lemma 2.8. Consider the diagram of spectra

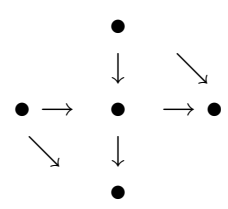

in which the row and the column are both cofibrations. Then the cofibres of the diagonal maps are naturally homotopy equivalent.

Proof. See [3].

Theorem 2.9. Let $\mathcal{X}$ be the filtration $Z \subset Y \subset X$ of topological manifolds, $n$ the dimension of $X, q$ the codimension of $Y$ in $X$, and $q^{\prime}$ the codimension of $Z$ in $Y$. Then there is a homotopy equivalence between the spectra

$$
\mathbb{L} T(X, Y, Z) \simeq \Sigma^{-q-q^{\prime}} \mathbb{L}^{B Q}(\mathcal{X}),
$$

and, consequently, an isomorphism

$$
L T_{n-q-q^{\prime}}(X, Y, Z)=L_{n}^{B Q}(\mathcal{X})
$$

between the surgery obstruction groups for $n=0,1,2,3 \bmod 4$.

Proof. It follows from Lemma 2.8 that the cofibres of the diagonal maps of spectra

$$
\begin{aligned}
\Sigma^{-q^{\prime}} \mathbb{L}\left(\pi_{1}(Y \backslash Z)\right) & \rightarrow \Sigma^{-q^{\prime}-q+1} \mathbb{L}\left(\pi_{1}(X \backslash Y)\right), \\
\mathbb{L} T(X, Y, Z) & \rightarrow \mathbb{L}\left(\pi_{1}(Z)\right) \\
\Sigma^{-q^{\prime}-q} \mathbb{L}\left(\pi_{1}(X \backslash Z)\right) & \rightarrow \Sigma^{-q^{\prime}+1} \mathbb{L} S\left(F_{Z}\right)
\end{aligned}
$$

in diagram (2.32) are naturally homotopy equivalent. The map of spectra

$$
\Sigma \mathbb{L}\left(\pi_{1}(Y \backslash Z)\right) \rightarrow \Sigma^{-q} \mathbb{L}\left(\pi_{1}(X \backslash Y)\right)
$$

is a realization at the spectrum level of the transfer map for the manifold pair $(X \backslash Z, Y \backslash Z)$ - this follows from diagram (2.28). Consequently, the cofibre of the first map in (2.33) coincides with the spectrum $\Sigma^{1-q^{\prime}} \mathbb{L} P\left(F_{Z}\right)$. Therefore it coincides with the cofibre of the second map in (2.33). We obtain the cofibration

$$
\mathbb{L} T(X, Y, Z) \rightarrow \mathbb{L}\left(\pi_{1}(Z)\right) \rightarrow \Sigma^{1-q^{\prime}} \mathbb{L} P\left(F_{Z}\right) .
$$

Consequently (see 33]), the spectrum $\mathbb{L} T(X, Y, Z)$ is defined as the homotopy fibre of the transfer map

$$
\mathbb{L}\left(\pi_{1}(Z)\right) \rightarrow \Sigma^{1-q^{\prime}} \mathbb{L} P\left(F_{Z}\right)
$$

But, according to (2.21), the homotopy fibre of this map is the spectrum $\Sigma^{-q-q^{\prime}} \mathbb{L}^{B Q}(\mathcal{X})$, where $\mathcal{X}$ is the filtration $Z \subset Y \subset X$. This proves the theorem. 
Corollary 2.10. Under the hypotheses of Theorem 2.9 we have the following three braids of exact sequences:

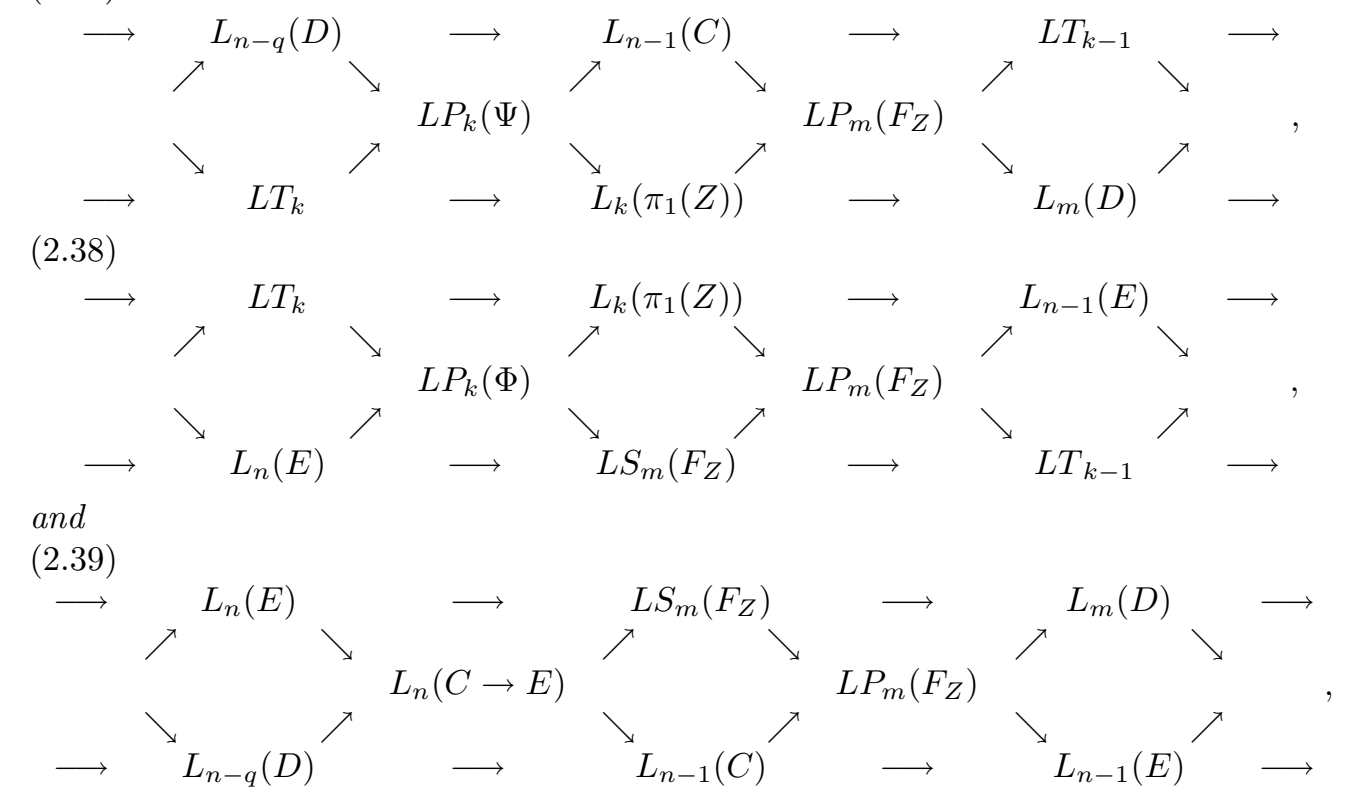

where $k=n-q-q^{\prime}, m=n-q-1, D=\pi_{1}(Y \backslash Z), E=\pi_{1}(X \backslash Z)$, and $C=\pi_{1}(X \backslash Y)$. Diagrams (2.37), (2.38), and (2.39) are realized at the spectrum level.

Proof. From the infinite homotopy commutative diagram (2.32) and the cofibration (2.35) we obtain the homotopy commutative diagram of spectra

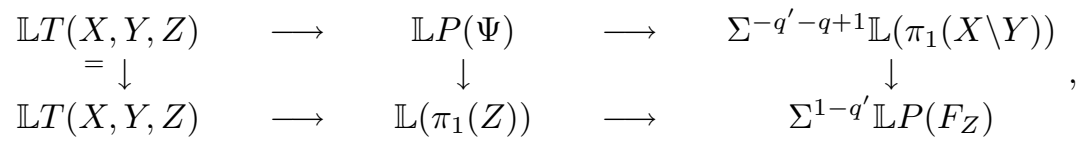

in which the horizontal rows are cofibrations and the right-hand vertical map is induced by the two left-hand vertical maps (see [33]). Consequently, the fibres of the two right-hand horizontal maps in (2.40) are naturally homotopy equivalent to the spectrum $\mathbb{L} T(X, Y, Z)$. Therefore the right-hand square in (2.40) is a pull-back square and the fibres of the vertical maps of this square are also naturally homotopy equivalent. The homotopy long exact sequences of this square give the commutative diagram (2.37). Similarly, the commutative diagrams (2.38) and (2.39) follow from the other two cofibrations in (2.33) and the homotopy commutative diagram (2.32).

Remark 2.11. Diagram (2.39) is in fact diagram (1.8) constructed for the pair of manifolds with boundary $(X \backslash Y) \subset(X \backslash Z)$.

Remark 2.12. We can regard the manifold pair $Y^{n-q} \subset X^{n}$ as a stratified manifold $\mathcal{X}$, for which the Browder-Quinn groups $L^{B Q}(\mathcal{X})$ are defined. It follows from the cofibration (2.12) that Wall's definition of the $L P_{*}$-groups and Ranicki's results (see [30, 34]) yield an isomorphism $L P_{n-q}(F) \cong L^{B Q}(\mathcal{X})$. This isomorphism is realized at the spectrum level.

\section{Surgery on a MANifold With a Filtration}

In this section we introduce the surgery obstruction groups for the filtration $\mathcal{X}$ in (1.11) and describe their basic properties. First we provide a motivation for our definition; then 
we prove Theorem 3.1 and describe the connection of the groups introduced here with the Browder-Quinn groups $L^{B Q}$. We use the notation of the preceding sections.

For a manifold pair $\left(X^{n}, Y^{n-q}\right)$ of codimension $q$ the realization of diagram (1.8) at the spectrum level gives rise to the homotopy commutative diagram of spectra

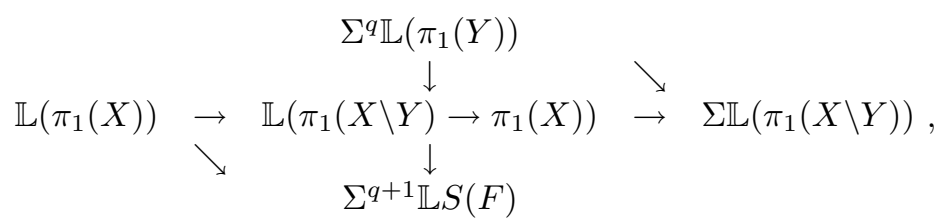

in which the vertical column and the horizontal row are cofibrations. The cofibres of the diagonal maps are naturally homotopy equivalent to the spectrum $\Sigma^{q+1} \mathbb{L} P(F)$; this follows from (1.8) and Lemma 2.8.

We now consider the manifold triple $Z^{n-q-q^{\prime}} \subset Y^{n-q} \subset X^{n}$, where $q$ is the codimension of $Y$ in $X$, and $q^{\prime}$ the codimension of $Z$ in $Y$. The realization of diagram (2.25) at the spectrum level gives rise to the homotopy commutative diagram of spectra

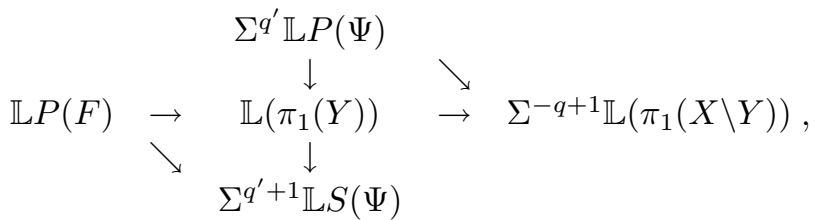

in which the vertical column and the horizontal row are cofibrations. Recall that $F$ is the square of fundamental groups in the splitting problem for the pair $(X, Y)$, while $\Psi$ is the analogous square for the pair $(Y, Z)$. Using (2.25) and Lemma 2.8, it follows that the cofibres of the diagonal maps are naturally homotopy equivalent to the spectrum $\Sigma^{q^{\prime}+1} \mathbb{L} T(X, Y, Z)$.

We now consider the filtration $\mathcal{X}$ in (1.11), for which the bounded filtrations $\mathcal{X}_{j}$ are defined for $j=0,1, \ldots, k$.

For a pair of submanifolds $X_{j} \subset X_{j-1}$ in the filtration (1.11), we let $F_{j}, 1 \leq j \leq k$, denote the square of fundamental groups in the splitting problem.

We also introduce a special notation for the following filtrations. Let $\mathcal{Y}$ be the subfiltration

$$
X_{k} \subset X_{k-1} \subset \cdots \subset X_{2} \subset X_{1}
$$

of the filtration $\mathcal{X}$, and $\mathcal{Y}_{j-1}$ the bounded subfiltration

$$
X_{j} \subset X_{j-1} \subset \cdots \subset X_{2} \subset X_{1}
$$

of the filtration $\mathcal{Y}$, where $1 \leq j \leq k$. We have $\mathcal{X}_{0}=\left(X_{0}\right)=(X), \mathcal{X}_{1}=\left(X_{1} \subset X_{0}\right)$, and $\mathcal{X}_{2}=\left(X_{2} \subset X_{1} \subset X_{0}\right)$. We set

$$
\begin{aligned}
& \mathbb{L} M^{0}(\mathcal{X})=\mathbb{L} M^{0}\left(X_{0}\right)=\mathbb{L}\left(\pi_{1}\left(X_{0}\right)\right), \\
& \mathbb{L} M^{1}(\mathcal{X})=\mathbb{L} M^{1}\left(\mathcal{X}_{1}\right)=\mathbb{L} M^{1}\left(X_{1} \subset X_{0}\right)=\mathbb{L} P\left(F_{1}\right),
\end{aligned}
$$

and

$$
\mathbb{L} M^{2}(\mathcal{X})=\mathbb{L} M^{2}\left(\mathcal{X}_{2}\right)=\mathbb{L} T\left(X_{0}, X_{1}, X_{2}\right) .
$$


By definition, $\mathbb{L} M^{i}\left(\mathcal{X}_{j}\right)=\mathbb{L} M^{i}(\mathcal{X})$ for the spectra $\mathbb{L} M^{i}$ defined above, where $0 \leq i \leq 2$ and $j \geq i$. In our notation, diagram (3.2) has the form

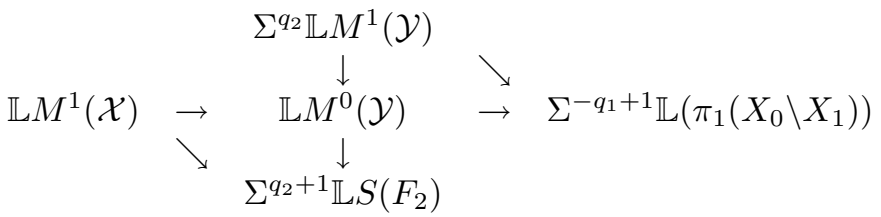

with the cofibres of the diagonal maps being naturally homotopy equivalent to

$$
\Sigma^{q_{2}+1} \mathbb{L} M^{2}(\mathcal{X})=\Sigma^{q_{2}+1} \mathbb{L} M^{2}\left(\mathcal{X}_{2}\right) .
$$

The right-hand diagonal map in (3.5) gives rise to the cofibration of spectra

$$
\mathbb{L} M^{2}(\mathcal{X}) \rightarrow \mathbb{L} M^{1}(\mathcal{Y}) \rightarrow \Sigma^{-q_{1}-q_{2}+1} \mathbb{L}\left(\pi_{1}\left(X_{0} \backslash X_{1}\right)\right),
$$

where $-q_{1}-q_{2}=l_{2}-n$. The left-hand diagonal map in (3.5) gives rise to the cofibration

$$
\Sigma^{q_{2}} \mathbb{L} M^{2}(\mathcal{X}) \rightarrow \mathbb{L} M^{1}(\mathcal{X}) \rightarrow \Sigma^{q_{2}+1} \mathbb{L} S\left(F_{2}\right) .
$$

For the filtration $\mathcal{Y}$, the cofibration (3.7) gives the cofibration

$$
\Sigma^{q_{3}} \mathbb{L} M^{2}(\mathcal{Y}) \rightarrow \mathbb{L} M^{1}(\mathcal{Y}) \rightarrow \Sigma^{q_{3}+1} \mathbb{L} S\left(F_{3}\right) .
$$

From the cofibrations (3.6) and (3.8) we can form the homotopy commutative diagram

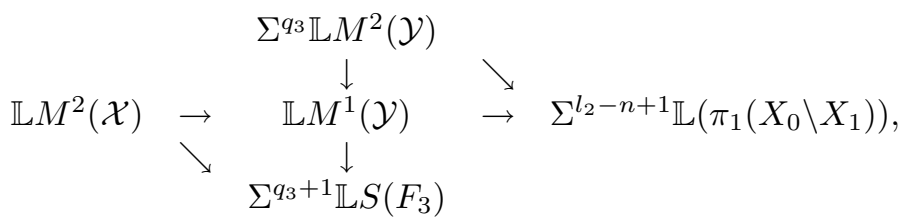

in which the cofibres of the diagonal maps are naturally homotopy equivalent. We denote the homotopy cofibre of the diagonal map in (3.9) by

$$
\Sigma^{q_{3}+1} \mathbb{L} M^{3}\left(\mathcal{X}_{3}\right)=\Sigma^{q_{3}+1} \mathbb{L} M^{3}(\mathcal{X}) .
$$

It follows from this definition that

$$
\mathbb{L} M^{3}\left(\mathcal{X}_{j}\right)=\mathbb{L} M^{3}(\mathcal{X}) \quad \text { for } 3 \leq j \leq k .
$$

We can extend these constructions so as to give an inductive definition of the spectrum

$$
\mathbb{L} M^{i}(\mathcal{X})=\mathbb{L} M^{i}\left(\mathcal{X}_{i}\right) \quad \text { for } 4 \leq i \leq k .
$$

Suppose that the spectrum $\mathbb{L} M^{j}(\mathcal{X})=\mathbb{L} M^{j}\left(\mathcal{X}_{j}\right)$ is already defined for $k \geq j \geq 2$ so that the spectrum $\Sigma^{q_{j}+1} \mathbb{L} M^{j}(\mathcal{X})$ (for $j \geq 2$ ) is the homotopy cofibre of the diagonal maps in the diagram

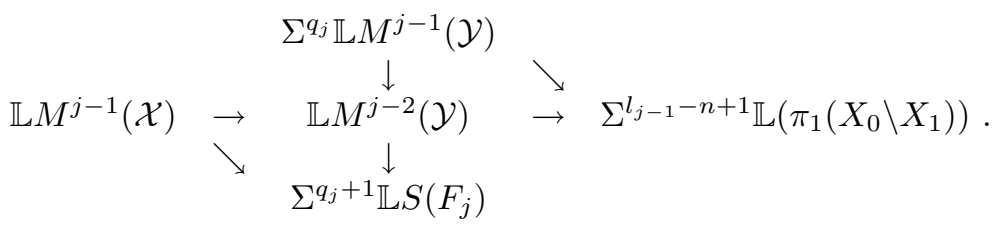

The right-hand diagonal map in (3.10) gives rise to the cofibration of spectra

$$
\mathbb{L} M^{j}(\mathcal{X}) \rightarrow \mathbb{L} M^{j-1}(\mathcal{Y}) \rightarrow \Sigma^{l_{j-1}-n-q_{j}+1} \mathbb{L}\left(\pi_{1}\left(X_{0} \backslash X_{1}\right)\right),
$$

where $l_{j-1}-n-q_{j}+1=l_{j}-n+1$. The left-hand diagonal map in (3.10) gives rise to the cofibration

$$
\Sigma^{q_{j}} \mathbb{L} M^{j}(\mathcal{X}) \rightarrow \mathbb{L} M^{j-1}(\mathcal{X}) \rightarrow \Sigma^{q_{j}+1} \mathbb{L} S\left(F_{j}\right)
$$


For the filtrations $\mathcal{Y}$ and $\mathcal{Y}_{j},(3.12)$ gives the cofibration

$$
\Sigma^{q_{j+1}} \mathbb{L} M^{j}(\mathcal{Y}) \rightarrow \mathbb{L} M^{j-1}(\mathcal{Y}) \rightarrow \Sigma^{q_{j+1}+1} \mathbb{L} S\left(F_{j+1}\right) .
$$

We can combine (3.11) and (3.13) to obtain the homotopy commutative diagram

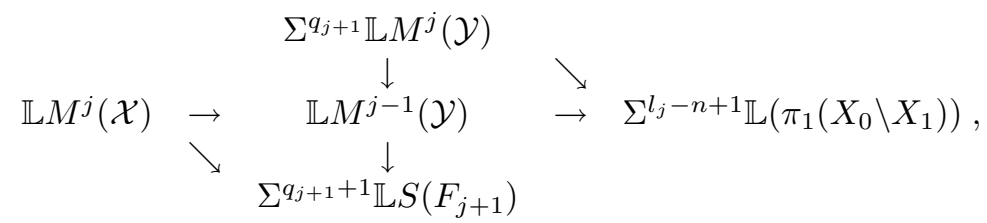

in which the cofibres of the diagonal maps are naturally homotopy equivalent. We denote the homotopy cofibre of the diagonal map in (3.14) by

$$
\Sigma^{q_{j+1}+1} \mathbb{L} M^{j+1}(\mathcal{X})=\Sigma^{q_{j+1}+1} \mathbb{L} M^{j}\left(\mathcal{X}_{j}\right) .
$$

Thus the spectra $\mathbb{L} M^{j}(\mathcal{X})$ are defined for $0 \leq j \leq k$. It follows from the definition that

$$
\mathbb{L} M^{j}(\mathcal{X})=\mathbb{L} M^{j}\left(\mathcal{X}_{i}\right) \quad \text { for } k \geq i \geq j .
$$

We define the groups $L M_{j}^{i}(\mathcal{X})$ to be the homotopy groups $\pi_{j}\left(\mathbb{L} M^{j}(\mathcal{X})\right)$. It follows from the definition that $j$ is defined mod 4 .

Proposition 3.1. Let $\mathcal{X}$ be the filtration (1.11). For $0 \leq j \leq k-2$ the groups LM occur in the braid of exact sequences

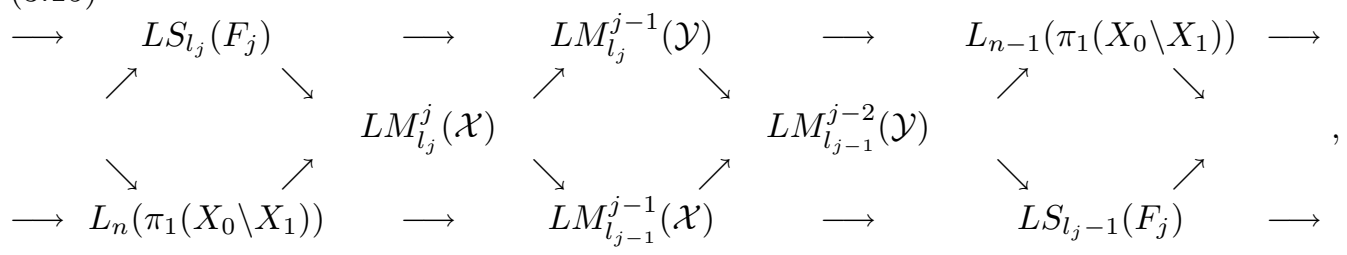

where $l_{j}$ is the dimension of the smallest manifold of the filtration. This diagram is realized at the spectrum level.

Proof. From the definition of $L M$-groups we obtain the homotopy commutative square of spectra

$$
\begin{array}{cccc}
\mathbb{L} M^{j-2}(\mathcal{Y}) & \rightarrow & \Sigma^{l_{j-1}-n+1} \mathbb{L}\left(\pi_{1}\left(X_{0} \backslash X_{1}\right)\right) \\
\downarrow & & \downarrow \\
\Sigma^{q_{j}+1} \mathbb{L} S\left(F_{j}\right) & \rightarrow & \Sigma^{q_{j}+1} \mathbb{L} M^{j}(\mathcal{X})
\end{array} .
$$

The fibres of the parallel maps in (3.16) are naturally homotopy equivalent - this follows from diagram (3.10). Consequently, the square (3.16) is a pull-back square and the consideration of the homotopy long exact sequences of maps in this square completes the proof of the proposition.

Corollary 3.2. For $2 \leq j \leq k$ the spectrum $\mathbb{L} M^{j}(\mathcal{X})$ occurs in the pull-back square of spectra

$$
\begin{array}{cccc}
\Sigma^{q_{j}} \mathbb{L} M^{j}(\mathcal{X}) & \rightarrow & \Sigma^{q_{j}} \mathbb{L} M^{j-1}(\mathcal{Y}) \\
\downarrow & & \downarrow \\
\mathbb{L} M^{j-1}(\mathcal{X}) & \rightarrow & \mathbb{L} M^{j-2}(\mathcal{Y})
\end{array} .
$$

We can now define the spectra for the structure sets of the filtration $\mathcal{X}$. According to Ranicki's definition [30] we define the spectrum $\mathbb{S}^{0}(\mathcal{X})=\mathbb{S}(X)$ for the manifold $X^{n}$ to be the homotopy cofibre of the map (1.4). 
For the filtration $\mathcal{X}$ that is defined by the manifold pair $\left(X^{n}, Y^{n-q}\right)$, the map

$$
H_{n}\left(X ; \mathbf{L}_{\bullet}\right) \rightarrow L P_{n-q}(F)
$$

in $(1.15)$ is realized at the spectrum level by the map

$$
X_{+} \wedge \mathbf{L}_{\bullet} \rightarrow \Sigma^{q} \mathbb{L} P(F)
$$

(see [1, 8, 29, 30]). We denote the cofibre of the map in $(3.18)$ by $\mathbb{S}^{1}(\mathcal{X})=\mathbb{S}(X, Y, \xi)$ with the homotopy groups

$$
\pi_{n}\left(\mathbb{S}^{1}(\mathcal{X})\right)=S_{n}(X, Y, \xi)
$$

occurring in the exact sequence (1.15).

For the filtration $\mathcal{X}=(Z \subset Y \subset X)$ we define the spectrum $\mathbb{S}^{2}(\mathcal{X})=\mathbb{S}(X, Y, Z)$ (see [8]) as the homotopy cofibre of the map

$$
X_{+} \wedge \mathbf{L}_{\bullet} \rightarrow \Sigma^{q+q^{\prime}} \mathbb{L} T(X, Y, Z) .
$$

Every $t$-triangulation of the filtration $\mathcal{X}$ in (1.11) gives a $t$-triangulation of the bounded filtrations $\mathcal{X}_{k}, \mathcal{Y}$, and $\mathcal{Y}_{k-1}$. Thus we obtain the commutative diagram

$$
\begin{array}{ccc}
\mathcal{T}(\mathcal{X}) & \rightarrow & \mathcal{T}(\mathcal{Y}) \\
\downarrow & & \downarrow \\
\mathcal{T}\left(\mathcal{X}_{k}\right) & \rightarrow & \mathcal{T}\left(\mathcal{Y}_{k}\right)
\end{array},
$$

which is realized at the spectrum level (see [8, 29, 30]). By Proposition 2.4 (see [30]), at the spectrum level diagram (3.20) has the form

$$
\mathcal{F}=\left(\begin{array}{ccc}
\left(X_{0}\right)_{+} \wedge \mathbf{L}_{\bullet} & \rightarrow & \Sigma^{q_{1}}\left[\left(X_{1}\right)_{+} \wedge \mathbf{L}_{\bullet}\right] \\
\downarrow & & \downarrow \\
\left(X_{0}\right)_{+} \wedge \mathbf{L}_{\bullet} & \rightarrow & \Sigma^{q_{1}}\left[\left(X_{1}\right)_{+} \wedge \mathbf{L}_{\bullet}\right]
\end{array}\right) .
$$

It follows from the definition of $\mathbb{L} M^{j}(\mathcal{X})$, together with (1.4), (3.18) and (3.19), that for $k \geq j \geq 0$ we have the maps

$$
\left(X_{0}\right)_{+} \wedge \mathbf{L} \bullet \rightarrow \Sigma^{n-l_{j}} \mathbb{L} M^{j}(\mathcal{X})
$$

whose cofibres we denote by $\mathbb{S}^{j}(\mathcal{X})$. Thus, $\mathbb{S}^{0}(\mathcal{X})=\mathbb{S}\left(X_{0}\right)$. Using the maps in (3.22) we obtain the map of squares

$$
\Lambda_{2}: \mathcal{F} \longrightarrow \mathcal{G}_{2},
$$

where

$$
\mathcal{G}_{2}=\left(\begin{array}{ccc}
\Sigma^{n-l_{2}} \mathbb{L} M^{2}(\mathcal{X}) & \rightarrow & \Sigma^{n-l_{2}} \mathbb{L} M^{1}(\mathcal{Y}) \\
\downarrow & & \downarrow \\
\Sigma^{n-l_{1}} \mathbb{L} M^{1}(\mathcal{X}) & \rightarrow & \Sigma^{n-l_{1}} \mathbb{L} M^{0}(\mathcal{Y})
\end{array}\right),
$$

which gives rise to a homotopy commutative diagram of spectra in the form of a cube. We note here that the square in (3.24) follows from (3.17).

The cofibres of the four maps that form the map $\Lambda_{2}$ give the pull-back square

$$
\begin{array}{ccc}
\mathbb{S}^{2}(\mathcal{X}) & \rightarrow & \Sigma^{q_{1}} \mathbb{S}^{1}(\mathcal{Y}) \\
\downarrow & & \downarrow \\
\mathbb{S}^{1}(\mathcal{X}) & \rightarrow & \Sigma^{q_{1}} \mathbb{S}^{0}(\mathcal{Y})
\end{array},
$$

since the squares (3.17) and (3.21) are pull-back squares.

Let $\mathcal{G}_{i}, k \geq i \geq 2$, be the homotopy commutative square of spectra

$$
\mathcal{G}_{i}=\left(\begin{array}{ccc}
\Sigma^{n-l_{i}} \mathbb{L} M^{i}(\mathcal{X}) & \rightarrow & \Sigma^{n-l_{i}} \mathbb{L} M^{i-1}(\mathcal{Y}) \\
\downarrow & & \downarrow \\
\Sigma^{n-l_{i-1}} \mathbb{L} M^{i-1}(\mathcal{X}) & \rightarrow & \Sigma^{n-l_{i-1}} \mathbb{L} M^{i-2}(\mathcal{Y})
\end{array}\right),
$$

which follows from (3.17). 
Proposition 3.3. For $k \geq i \geq 2$ there exist maps of squares

$$
\Lambda_{i}: \mathcal{F} \longrightarrow \mathcal{G}_{i}
$$

that are defined by four maps so that the resulting diagram in the form of a cube is homotopy commutative.

Proof. Using induction on $i$ it is sufficient to define the left-hand upper map in $\Lambda_{i}$ when the other three maps have already been defined. This is possible, since the homotopy commutative square (3.26) is a pull-back square.

Definition. Let $\mathcal{X}$ be the filtration (1.11). For $k \geq j \geq 0, \mathbb{S}^{j}(\mathcal{X})$ denotes the homotopy cofibre of the map

$$
\left(X_{0}\right)_{+} \wedge \mathbf{L} \bullet \rightarrow \Sigma^{n-l_{j}} \mathbb{L} M^{j}(\mathcal{X})
$$

that is defined by the map $\Lambda_{i}$ in (3.27). The homotopy groups $\pi_{n}\left(\mathbb{S}^{j}(\mathcal{X})\right)$ are denoted by $\mathcal{S}_{n}^{j}(\mathcal{X})$.

The structure sets $\mathcal{S}_{n}^{j}(\mathcal{X})$ are natural generalizations of the structure set $\mathcal{S}_{n}(X)$ of homotopy triangulations of the manifold $X$ and of the set $\mathcal{S}_{n}(X, Y, \xi)$ of homotopy triangulations of the manifold pair. We now describe the basic properties of these sets.

Remark 3.4. Let $\mathcal{X}$ be the filtration (1.11). For $k \geq j \geq 0$ it follows from the definition that we have the exact sequence

$$
\cdots \rightarrow \mathcal{S}_{n+1}^{j}(\mathcal{X}) \rightarrow H_{n}\left(X ; \mathbf{L}_{\bullet}\right) \rightarrow L M_{l_{j}}^{j}(\mathcal{X}) \rightarrow \cdots
$$

For $j=0$ and $X=X_{0}$ the exact sequence (3.29) coincides with (1.2), for $j=1$ it coincides with (1.15) for the pair $X_{1} \subset X_{0}$, and for $j=2$ it coincides with the homotopy long exact sequence of the cofibration (3.19) for the triple $X_{2} \subset X_{1} \subset X_{0}$.

Proposition 3.5. For $k \geq i \geq 2$ there exists the following homotopy commutative pullback square of spectra:

$$
\begin{array}{cccc}
\mathbb{S}^{i}(\mathcal{X}) & \rightarrow & \Sigma^{q_{1}} \mathbb{S}^{i-1}(\mathcal{Y}) \\
\downarrow & & \downarrow \\
\mathbb{S}^{i-1}(\mathcal{X}) & \rightarrow & \Sigma^{q_{1}} \mathbb{S}^{i-2}(\mathcal{Y})
\end{array} .
$$

Proof. The square (3.30) is obtained as the square of the homotopy cofibres of the maps that constitute the map $\Lambda_{i}$. The squares (3.21) and (3.27) are pull-back squares. Consequently, the square (3.30) is a pull-back square.

Corollary 3.6. For $k \geq i \geq 2$ there exists the following braid of exact sequences:

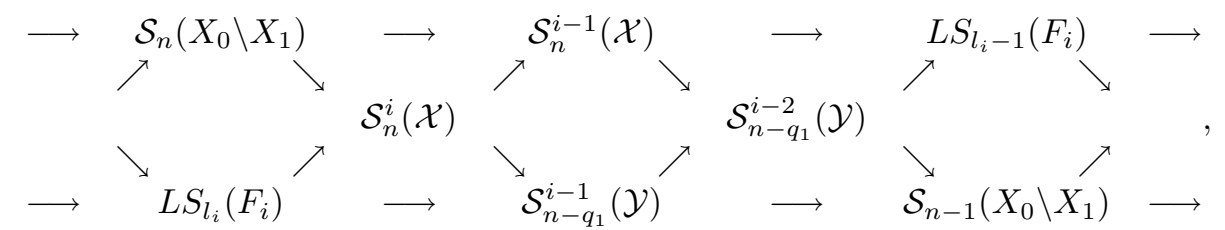

where $\mathcal{S}_{n}\left(X_{0} \backslash X_{1}\right)$ is the structure set occurring in (1.2), the algebraic exact sequence of surgery theory for $X_{0} \backslash X_{1}$.

Proof. The homotopy long exact sequences of the maps in the pull-back square (3.30) give the diagram (3.31).

In the case of manifold triples, diagram (3.31) was obtained in 8 . In fact, this diagram is a natural generalization of the diagram in [30, Proposition 7.2.6 ii)], which holds for manifold pairs. 
Proposition 3.7. For $k \geq i \geq 1$ we have the following homotopy commutative pull-back square of spectra:

$$
\begin{array}{cccc}
\Sigma^{-1} \mathbb{S}^{i-1}(\mathcal{X}) & \rightarrow & \left(X_{0}\right)_{+} \wedge \mathbf{L}_{\bullet} \\
\downarrow & & \downarrow \\
\Sigma^{n-l_{i}} \mathbb{L} S\left(F_{i}\right) & \rightarrow & \Sigma^{n-l_{i}} \mathbb{L} M^{i}(\mathcal{X})
\end{array} .
$$

Proof. We have the homotopy commutative diagram

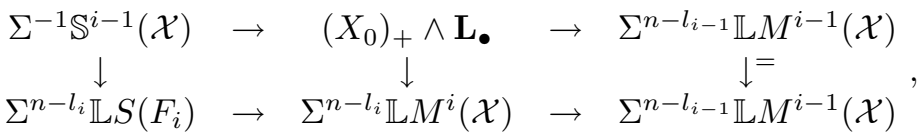

where the right-hand square follows from Proposition 3.3, and the left-hand map is obtained as the natural map of the fibres of the horizontal maps of the right-hand square 33. Using the definition of the spectrum $\mathbb{S}^{i}(\mathcal{X})$ and Corollary 3.6, the fibres have this property. Now the cofibres of the horizontal maps of the left-hand square in (3.33) are naturally homotopy equivalent, and this square is a push-out square and therefore also a pull-back square.

Corollary 3.8. For $k \geq i \geq 1$ we have the following braid of exact sequences:

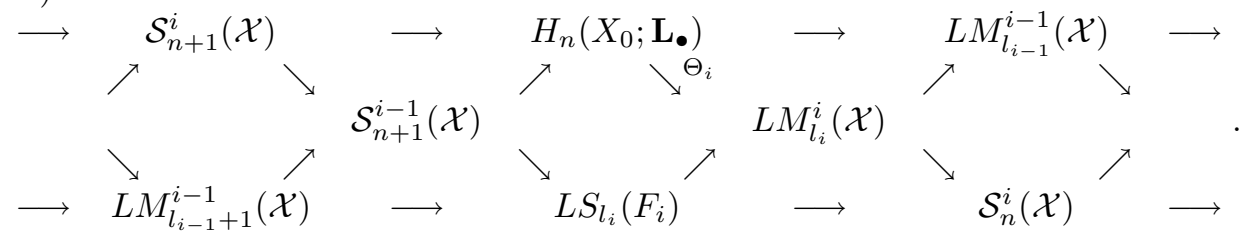

Proof. The homotopy long exact sequence of the maps in the pull-back square (3.32) gives the commutative diagram of exact sequences (3.31).

The diagram (3.34) for the case of a manifold pair was obtained in 30, Proposition 7.2.6 iv)]. For the case of a manifold triple this diagram was obtained in [8, Theorem 4].

Theorem 3.9. Let $\mathcal{X}$ be the filtration (1.11), where the dimension of the submanifold $X_{k}$ is equal to $l_{k} \geq 5$, and let

$$
x=(f, b) \in\left[X_{0}, G / T O P\right]=H_{n}\left(X ; \mathbf{L}_{\bullet}\right)
$$

be some $t$-triangulation of $\mathcal{X}$ with a given map $f: M \rightarrow X=X_{0}$. Then the map $(f, b)$ is normally bordant to an s-triangulation of the filtration $\mathcal{X}$ if and only if $\Theta_{k}(x)=0$. The set $\mathcal{S}_{n+1}^{i}(\mathcal{X})$ can be identified with the set of the concordance classes of s-triangulations of $\mathcal{X}_{i}$.

Proof. We use induction on $k$, the number of submanifolds. For $k=1,2$ the result was obtained in [8, 30, respectively.

Let $x=[(f, b)] \in H_{n}\left(X_{0} ; \mathbf{L}_{\bullet}\right)$ be an $s$-triangulation of the filtration $\mathcal{X}_{k}$. It follows from the definition that $x$ is an $s$-triangulation of the subfiltration $\mathcal{X}_{k-1}$ such that the restriction to $X_{k-1}$ is already split along the submanifold $X_{k} \subset X_{k-1}$. Consequently, $\Theta_{i-1}(x)=0$ by the induction hypothesis, and it follows from (3.34) that $x$ represents an element $y \in \mathcal{S}_{n+1}^{k-1}\left(\mathcal{X}_{k-1}\right)$. It follows from diagram (3.31) that the map

$$
\sigma: \mathcal{S}_{n+1}^{k-1}(\mathcal{X})=\mathcal{S}^{k-1}\left(\mathcal{X}_{k-1}\right) \rightarrow L S_{l_{k}}\left(F_{k}\right)
$$

in (3.34) is defined by the composite

$$
\mathcal{S}_{n+1}^{k-1}\left(\mathcal{X}_{k-1}\right) \rightarrow \mathcal{S}_{l_{k-1}+1}\left(X_{k-1}\right) \rightarrow L S_{l_{k}}\left(F_{k}\right) .
$$


The right-hand map in (3.35) is the map in diagram (1.7) for the pair $\left(X_{k-1}, X_{k}\right)$. Since the restriction of $x$ to $X_{k-1}$ splits along $X_{k}$, according to the geometric definition of the map $\mathcal{S}_{l_{k-1}+1}\left(X_{k-1}\right) \rightarrow L S_{l_{k}}\left(F_{k}\right)$ we obtain $\sigma(y)=0$. It now follows from (3.34) that $\Theta_{i}(x)=0$.

We now prove the converse implication. Suppose that $\Theta_{k}(x)=0$. It follows from diagram (3.34) that $\Theta_{k-1}(x)=0$. Consequently, there exists an element $y \in \mathcal{S}_{n+1}^{k-1}(\mathcal{X})$ that is mapped to $x$. The set $\mathcal{S}_{n+1}^{k-1}(\mathcal{X})$ can be identified with the concordance classes of $s$-triangulations of the filtration $\mathcal{X}_{k-1}$. Therefore a representative of $y$ gives rise to an $s$-triangulation $\left(f^{\prime}, b^{\prime}\right)$ from $\mathcal{X}_{k-1}$. Since $\Theta_{k}(x)=0$ and (3.34) is commutative, thus $y$ belongs to the image of the map

$$
\mathcal{S}_{n+1}^{k}(\mathcal{X}) \rightarrow \mathcal{S}_{n+1}^{k-1}(\mathcal{X})
$$

in (3.34). This means that $\sigma(x)=0$ and, according to the factorization (3.35), the restriction of the map $f^{\prime}$ to $X_{k-1}$ splits along the submanifold $X_{k}$. We can extend the homotopy to obtain an $s$-triangulation of $\mathcal{X}_{k-1}$ whose restriction to $X_{k-1}$ is an $s$-triangulation of the pair $\left(X_{k}, X_{k-1}\right)$. An application of Proposition 2.5 completes the proof of the theorem.

We now describe the relations between the surgery obstruction groups $L M_{*}^{i}(\mathcal{X})=$ $L M^{i}\left(\mathcal{X}_{i}\right)$ for the filtration (1.11) introduced above and the Browder-Quinn stratified $L$-groups $L_{*}^{B Q}\left(\mathcal{X}_{i}\right)$ (see [14, 35]).

The Browder-Quinn groups of the filtration $\mathcal{X}$ are realized at the spectrum level, and we recall here the inductive definition of these groups given in [35, p. 129], using our notation. In accordance with Theorem 2.9 we have the homotopy equivalence

$$
\mathbb{L} M^{2}(\mathcal{X}) \simeq \Sigma^{l_{2}-n} \mathbb{L}^{B Q}\left(\mathcal{X}_{2}\right)
$$

It should be pointed out that in a similar fashion the homotopy equivalence of spectra

$$
\mathbb{L} M^{1}(\mathcal{X})=\mathbb{L} P\left(F_{1}\right) \simeq \Sigma^{l_{1}-n} \mathbb{L}^{B Q}\left(\mathcal{X}_{1}\right)
$$

immediately follows from (2.10), (2.12), and the definition in [14, p. 129].

According to Remark 2.3 the filtration $\mathcal{X}$ gives rise to the filtration of manifolds with boundary $\overline{\mathcal{X}}$. The boundaries of the latter filtration give the filtration of closed manifolds

$$
\partial\left(X_{k-1} \backslash X_{k}\right) \subset \partial\left(X_{k-2} \backslash X_{k}\right) \subset \cdots \subset \partial\left(X_{1} \backslash X_{k}\right) \subset \partial\left(X_{0} \backslash X_{k}\right),
$$

which we denote by $\partial \overline{\mathcal{X}}$. Note that the filtrations $\partial \overline{\mathcal{X}}$ and $\overline{\mathcal{X}}$ contain $k$ spaces each, and the filtration $\mathcal{X}$ contains $k+1$ spaces.

We consider the homotopy commutative diagram of spectra

$$
\begin{array}{ccccccc}
\mathbb{L} M^{j}(\overline{\mathcal{X}}) & \rightarrow & \mathbb{L} M^{j-1}(\overline{\mathcal{Y}}) & \rightarrow & \Sigma^{l_{j}-n+1} \mathbb{L}(C) & \rightarrow & \Sigma \mathbb{L} M^{j}(\overline{\mathcal{X}}) \\
\downarrow & & \downarrow & & \downarrow= & & \downarrow \\
\mathbb{L} M^{j}(\mathcal{X}) & \rightarrow & \mathbb{L} M^{j-1}(\mathcal{Y}) & \rightarrow & \Sigma^{l_{j}-n+1} \mathbb{L}(C) & \rightarrow & \Sigma \mathbb{L} M^{j}(\mathcal{X})
\end{array},
$$

where $j=k-1 \geq 1, C=\pi_{1}\left(X_{0} \backslash X_{1}\right)$, and the horizontal rows are cofibrations according to (3.14). The vertical maps in (3.38) are induced by the natural embeddings of the filtrations $\overline{\mathcal{X}_{k}} \subset \mathcal{X}_{k-1}$. For $k-1=j=1$ the central square in (3.38) is involved in the homotopy commutative diagram of spectra

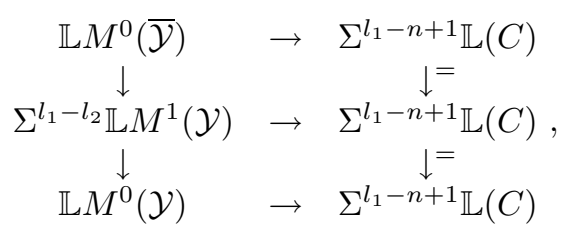

which follows from diagrams (3.14) and (1.8) at the spectrum level for the pair $\left(X_{1}, X_{2}\right)$. 
Proposition 3.10. Let $\mathcal{X}$ be the filtration (1.11) with $k \geq 2$. We have the following commutative diagram of spectra:

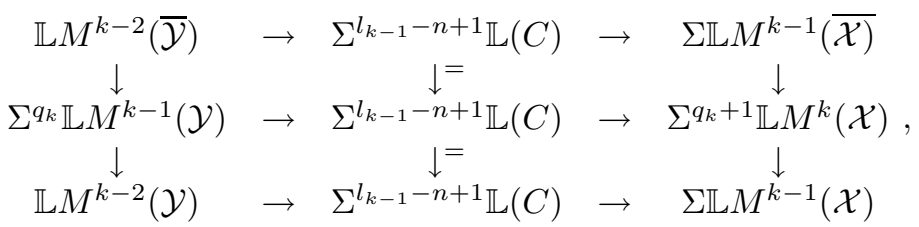

where $l_{k-1}-l_{k}=q_{k}$ and $C=\pi_{1}\left(X_{0} \backslash X_{1}\right)$. The right-hand vertical composite coincides with the right-hand vertical map in diagram (3.38) for $j=k-1$.

Proof. For $k=2$ the result follows from (3.39) and (3.38) if we define the right-hand vertical maps in (3.40) to be the natural maps of the homotopy cofibres of the corresponding horizontal maps in (3.39) (see [33]). Induction on $k$ now completes the proof of the proposition.

Corollary 3.11. Let $\mathcal{X}$ be the filtration (1.11) with $k \geq 1$. Then the homotopy fibre of the map

$$
\mathbb{L} M^{k-1}(\overline{\mathcal{X}}) \rightarrow \Sigma^{q_{k}} \mathbb{L} M^{k}(\mathcal{X})
$$

is naturally homotopy equivalent to $\Sigma^{q_{k}-1} \mathbb{L}\left(\pi_{1}\left(X_{k}\right)\right)$.

Proof. For $k=1$ the result follows from the definition of the spectra $\mathbb{L} M^{0}, \mathbb{L} M^{1}=$ $\mathbb{L} P\left(F_{1}\right)$ and the cofibration (2.12). For $k \geq 2$ the result follows by induction from the homotopy commutative diagram

$$
\begin{aligned}
& \Sigma^{l_{k-1}-n} \mathbb{L}(C) \quad \rightarrow \quad \mathbb{L} M^{k-1}(\overline{\mathcal{X}}) \quad \rightarrow \quad \mathbb{L} M^{k-2}(\overline{\mathcal{Y}}) \\
& \begin{array}{c}
\downarrow= \\
\Sigma^{l_{k-1}-n} \mathbb{L}(C) \rightarrow \Sigma^{q_{k}} \stackrel{\downarrow}{L} M^{k}(\mathcal{X}) \rightarrow \Sigma^{q_{k}} \mathbb{L} M^{k-1}(\mathcal{Y})
\end{array},
\end{aligned}
$$

which follows from (3.40). The right-hand square in (3.42) is a pull-back square, since the fibres of the horizontal maps are naturally homotopy equivalent. Consequently, the fibres of the vertical maps are also naturally homotopy equivalent. By the induction hypothesis the fibre of the right-hand vertical map is homotopy equivalent to $\Sigma^{q_{k}-1} \mathbb{L}\left(\pi_{1}\left(X_{k}\right)\right)$. This proves the corollary.

We now recall that in [35, p. 129] an inductive definition of the spectrum $\mathbb{L}^{B Q}(\mathcal{X})$ was given, with homotopy groups

$$
\pi_{n}\left(\mathbb{L}^{B Q}(\mathcal{X})\right)=L_{n}^{B Q}(\mathcal{X})
$$

which are Browder-Quinn L-groups [14].

Proposition 3.12. Let $\mathcal{X}$ be the filtration (1.11), where the smallest manifold $X_{k}$ has dimension $l_{k}$. Then there is a natural homotopy equivalence

$$
\mathbb{L} M^{k}(\mathcal{X}) \simeq \Sigma^{l_{k}-n} \mathbb{L}^{B Q}(\mathcal{X}) .
$$

Proof. For $k \geq 1$, the cofibration (3.41) gives the cofibration

$$
\mathbb{L}\left(\pi_{1}\left(X_{k}\right)\right) \rightarrow \Sigma^{1-q_{k}} \mathbb{L} M^{k-1}(\overline{\mathcal{X}}) \rightarrow \Sigma^{1} \mathbb{L} M^{k}(\mathcal{X}),
$$

which coincides with the cofibration in the inductive definition of the spectrum $\mathbb{L}^{B Q}(\mathcal{X})$ [35. p. 129] up to a shift in the numbering of the spaces of the spectrum. 
Proposition 3.13. Let $\mathcal{X}$ be the filtration (1.11) with $k \geq 2$. We have the braid of exact sequences

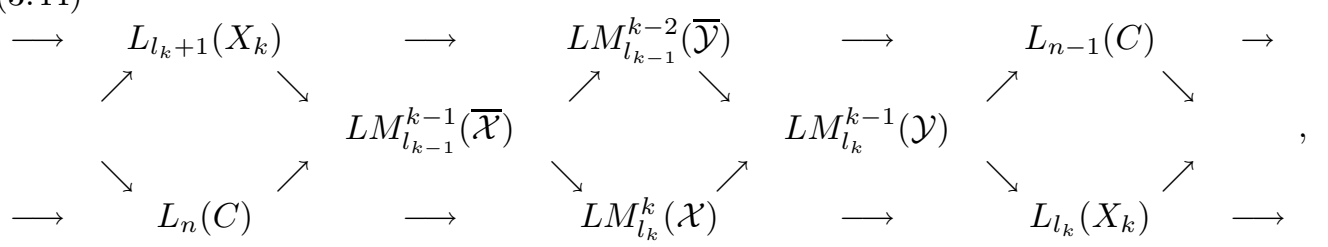

where $C=\pi_{1}\left(X_{0} \backslash X_{1}\right)$.

Proof. The homotopy long exact sequence of the maps in the right-hand pull-back square in diagram (3.42) gives rise to the commutative braid of exact sequences (3.44).

\section{Application to the Browder-Livesay invariants}

The filtration $\mathcal{X}$ given by (1.11) is called a Browder-Livesay filtration if for each $k \geq j \geq 1$ the manifold pair $X_{j} \subset X_{j-1}$ is a Browder-Livesay pair. Note that $F_{j}, 1 \leq$ $j \leq k$, is the square of fundamental groups in the splitting problem for the manifold pair $X_{j} \subset X_{j-1}$ in the filtration (1.11). For a Browder-Livesay filtration each submanifold $X_{j}$ is a one-sided submanifold of codimension 1 in $X_{j-1}$, the horizontal maps in the squares $F_{j}$ are isomorphisms, and the vertical maps are embeddings of index 2.

Theorem 4.1. Let $\mathcal{X}$ be a Browder-Livesay filtration (1.11) in which all the squares $F_{j}$ for $1 \leq j \leq k$ are the same. Then the filtration of spectra (1.14) has the form

$$
\Sigma^{k} \mathbb{L} M^{k} \rightarrow \Sigma^{k-1} \mathbb{L} M^{k-1} \rightarrow \cdots \rightarrow \Sigma^{1} \mathbb{L} M^{1} \rightarrow \mathbb{L} M^{0}
$$

and coincides with the left-hand side, starting from $\mathbb{X}_{0,0}$, of the filtration (1.10) for the Hambleton-Kharshiladze spectral sequence.

Proof. An inductive definition of the spectrum $\mathbb{L} M^{j}(\mathcal{X})$ follows from Corollary 3.2. This spectrum is constructed by using the three other spectra in diagram (3.17) so as to obtain a pull-back square. But the spectrum $\mathbb{X}_{j, 0}$ of the filtration (1.10) is defined inductively by using the same construction (see [8, 12]).

Let $i_{+}: A \rightarrow B^{+}$be an embedding of groups of index 2 defining the square (1.9). For such an embedding Ranicki 31] constructed an algebraic version of diagram (1.8):

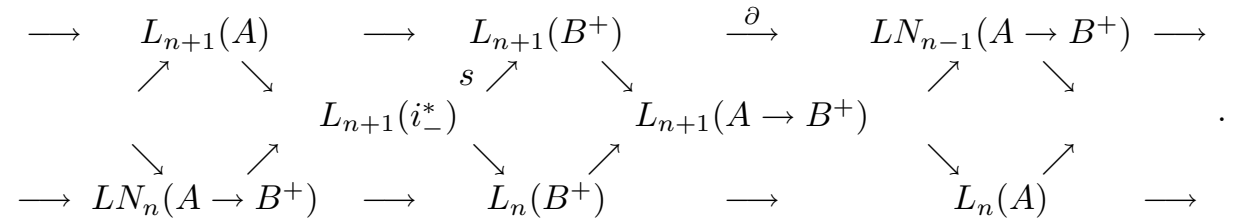

For a Browder-Livesay pair $Y \subset X$ with the square (1.9) of fundamental groups, diagram (1.8) coincides with (4.2). The map $\partial$ defines the Browder-Livesay invariant. If $\partial(x) \neq 0$, then the element $x \in L_{n+1}(B)$ cannot be realized by a normal map of closed manifolds (see [20]).

Diagram (4.2) is realized at the spectrum level, and we can write down the pull-back square of spectra

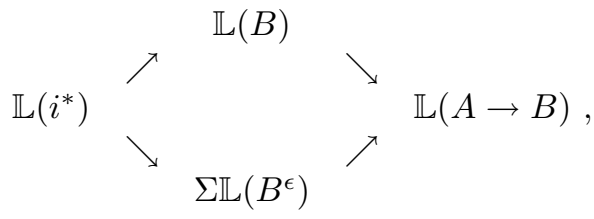


where $i^{*}$ denotes $i_{-}^{*}$, while $B^{\epsilon}$ means that the orientation on the lower group $B$ differs from the orientation on the upper group $B$ outside the image of the map $i: A \rightarrow B$. We consider the sequence $\mathcal{A}$ of embeddings of subgroups of index 2 into the group $B$ with orientation

$$
i_{1}: A_{1} \rightarrow B ; \quad i_{2}: A_{2} \rightarrow B^{\epsilon_{1}} ; \ldots ; \quad i_{k}: A_{k} \rightarrow B^{\epsilon_{k-1}} ; \ldots
$$

in which the orientation of the group $B^{\epsilon_{i}}$ coincides with the orientation of $B^{\epsilon_{i-1}}$ on the image of $A_{i} \rightarrow B$, and differs from it outside this image. Each embedding in (4.4) gives rise to a pull-back square analogous to (4.3) and we can write down the column of pull-back squares

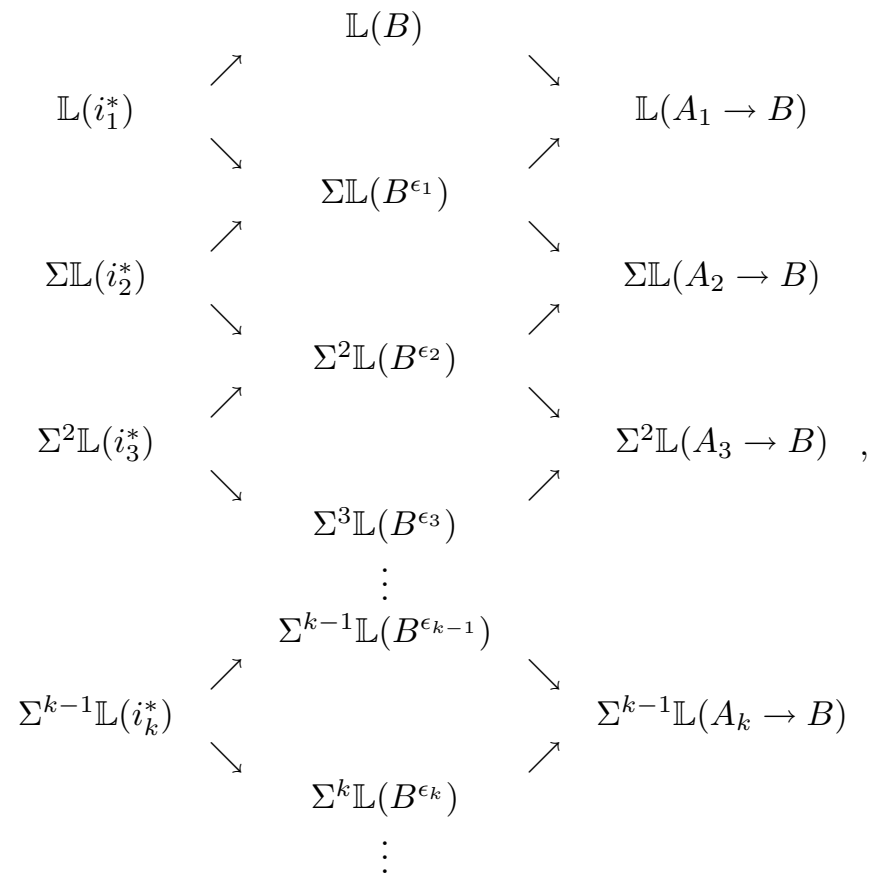

which is subject to the same convention on the orientations as the square (4.3).

Let $\mathbb{L} M^{0}(\mathcal{A})=\mathbb{L}(B)$ and $\Sigma \mathbb{L} M^{1}(\mathcal{A})=\mathbb{L}\left(i_{1}^{*}\right)$. Using the construction of a pull-back square we can, similarly to [12, extend the diagram (4.5) to the left. In particular, we obtain the filtration of spectra

$$
\cdots \rightarrow \Sigma^{k} \mathbb{L} M^{k}(\mathcal{A}) \rightarrow \Sigma^{k-1} \mathbb{L} M^{k-1}(\mathcal{A}) \rightarrow \cdots \rightarrow \Sigma^{1} \mathbb{L} M^{1}(\mathcal{A}) \rightarrow \mathbb{L} M^{0}(\mathcal{A}),
$$

which is the left-hand oblique row in the extended diagram.

We use the filtration (4.6) to construct the spectral sequence

$$
E_{r}^{p, q}=E_{r}^{p, q}(\mathcal{A})
$$

for the sequence of embeddings $\mathcal{A}$, similarly to 12 . We define the first term

$$
E_{1}^{p, q}=\pi_{q-p}\left(\Sigma^{p} \mathbb{L} M^{p}(\mathcal{A}), \Sigma^{p+1} \mathbb{L} M^{p+1}(\mathcal{A})\right) \cong L N_{q-2 p-2}\left(A_{p} \rightarrow B^{\epsilon_{p}}\right)
$$

and the first differential

$$
d_{1}^{p, q}: E_{1}^{p, q} \rightarrow E_{1}^{p+1, q}
$$

that coincides with the composite

$$
L N_{q-2 p-2}\left(A_{p+1} \rightarrow B^{\epsilon_{p}}\right) \rightarrow L_{q-2 p-2}\left(B^{\epsilon_{p+1}}\right) \rightarrow L N_{q-2 p}\left(A_{p+2} \rightarrow B^{\epsilon_{p+1}}\right) .
$$

The first map of the composite (4.8) occurs in diagram (4.3) for the embedding

$$
A_{p+1} \rightarrow B^{\epsilon_{p}}
$$


and the second map occurs in the same diagram for the embedding

$$
A_{p+2} \rightarrow B^{\epsilon_{p+1}}
$$

(see [12]). Note that the sequence thus obtained is a trivial generalization of the spectral sequence constructed in [12. The general results in [12] on the spectral sequence in surgery theory can be applied to the spectral sequence obtained above.

Remark 4.2. Suppose that in (4.4) all the embeddings $A_{i} \rightarrow B$ coincide with $A \rightarrow B$. Then the spectral sequence constructed above coincides with the spectral sequence in 12 ] for the embedding $A \rightarrow B$ of index 2 .

We observe that a finite sequence $\mathcal{A}$ of embeddings $i_{j}, 1 \leq j \leq k$, as in (4.4), gives rise to the finite filtration of spectra

$$
\Sigma^{k} \mathbb{L} M^{k}(\mathcal{A}) \rightarrow \Sigma^{k-1} \mathbb{L} M^{k-1}(\mathcal{A}) \rightarrow \cdots \rightarrow \Sigma^{1} \mathbb{L} M^{1}(\mathcal{A}) \rightarrow \mathbb{L} M^{0}(\mathcal{A}) .
$$

The Browder-Livesay filtration $\mathcal{X}$ in (1.11) gives the finite sequence of squares

$$
F_{j}=\left(\begin{array}{ccc}
A_{j} & \cong & A_{j} \\
\downarrow^{i_{-}} & & \downarrow^{i_{+}} \\
B^{\epsilon_{j}} & \cong & B^{\epsilon_{j-1}}
\end{array}\right)
$$

of fundamental groups for $1 \leq j \leq k$, which is analogous to (1.9). The right-hand vertical embeddings in (4.10) give a finite sequence $\mathcal{A}(\mathcal{X})$ of embeddings of index 2 into the group $B$.

Proposition 4.3. Under these assumptions we have

$$
\mathbb{L} M^{j}(\mathcal{X})=\mathbb{L} M^{j}(\mathcal{A}(\mathcal{X}))
$$

for $1 \leq j \leq k$.

Proof. The proof is the same as the proof of Theorem 4.1.

For the sequence of embeddings $\mathcal{A}$ in (4.4) we can construct the filtration of spectra (4.6). We denote the homotopy groups of the spectra in the filtration as follows:

$$
\pi_{n}\left(\mathbb{L} M^{k}(\mathcal{A})\right)=L M_{n}^{k}(\mathcal{A}) .
$$

Thus the filtration (4.6) gives rise to the tower of groups

$$
\cdots \rightarrow L M_{n-j}^{j}(\mathcal{A}) \rightarrow L M_{n-j+1}^{j-1}(\mathcal{A}) \rightarrow \cdots \rightarrow L M_{n-1}^{1}(\mathcal{A}) \rightarrow L M_{n}^{0}(\mathcal{A})=L_{n}(B)
$$

We denote by $\phi_{j}$ the map

$$
L M_{n-j}^{j}(\mathcal{A}) \rightarrow L M_{n}^{0}(\mathcal{A})=L_{n}(B),
$$

which is the composite of the maps in (4.11). The map $\phi_{1}$ is the map $s$ in diagram (4.2).

Theorem 4.4. Suppose that an element $x \in L_{n}(B)$, where $n$ is given $\bmod 4$, does not belong to the image of $\phi_{j}$ for some sequence of embeddings $\mathcal{A}$ and some positive integer $j$. Then $x$ cannot be realized by a normal map of closed manifolds.

Proof. Suppose that an element $x \in L_{n}(B)$ is realized by a normal map of closed manifolds $(f, b): M^{n} \rightarrow X^{n}$. According to [34, $\left.\S 9\right]$ multiplication by the complex projective space $P_{2}(\mathbb{C})$ of dimension 4 gives rise to the problem of surgery theory

$$
f \times I d: M^{n} \times P_{2}(\mathbb{C}) \rightarrow X^{n} \times P_{2}(\mathbb{C})
$$

in dimension $n+4$ with surgery obstruction $x \in L_{n}(B)$. Iterating this construction we obtain a normal map of closed manifolds

$$
g=f \times I d: M \times\left(P_{2}(\mathbb{C})\right)^{k} \rightarrow X \times\left(P_{2}(\mathbb{C})\right)^{k}=X_{0}
$$


in dimension $m=n+4 k \geq j+5$ with surgery obstruction $\Theta_{0}\left(g, b^{\prime}\right)=x \in L_{n}(B)$. Let $\mathcal{A}$ denote the sequence of embeddings

$$
i_{1}: A_{1} \rightarrow B ; \quad i_{2}: A_{2} \rightarrow B ; \ldots ; \quad i_{j}: A_{j} \rightarrow B
$$

that defines the map $\phi_{j}$. We consider a map

$$
\psi_{1}: X_{0} \rightarrow P_{N}(\mathbb{R})
$$

that induces an epimorphism of the fundamental groups with kernel $A_{1}$. Here $P_{N}(\mathbb{R})$ is a real projective space of high dimension. Varying the map $\psi_{1}$ in its homotopy class we can assume that $\psi_{1}$ is transversal to $P_{N-1}(\mathbb{R}) \subset P_{N}(\mathbb{R})$ with

$$
\psi_{1}^{-1}\left(P_{N-1}(\mathbb{R})\right)=X_{1}
$$

and that $X_{1} \subset X_{0}$ is a Browder-Livesay pair (see [10, 12, 16, 21]). In similar fashion we can now consider a map

$$
\psi_{2}: X_{1} \rightarrow P_{N}(\mathbb{R})
$$

that induces an epimorphism of the fundamental groups with kernel $A_{2}$ and with

$$
\psi_{2}^{-1}\left(P_{N-1}(\mathbb{R})\right)=X_{2},
$$

where $X_{2} \subset X_{1}$ is a Browder-Livesay pair. Iterating this construction we obtain a Browder-Livesay filtration $\mathcal{X}$

$$
X_{j} \subset X_{j-1} \subset \cdots \subset X_{1} \subset X_{0}
$$

with $\mathcal{A}(\mathcal{X})=\mathcal{A}$. From Corollary 3.8 we obtain the commutative diagram

$$
H_{m}\left(\underset{\left.X_{0} ; \mathbf{L}_{\bullet}\right)}{\mathbf{L}_{\bullet}} \stackrel{\Theta_{0}}{\longrightarrow} \underset{\phi_{j} \nearrow}{L_{m}(B)}{ }_{\phi_{n-j}^{j}}\right.
$$

It follows from (4.15) that

$$
\phi_{j} \Theta_{j}\left(g, b^{\prime}\right)=\Theta_{0}\left(g, b^{\prime}\right)=x \in L_{m}(B),
$$

and therefore the element $x$ belongs to the image of $\phi_{j}$. We have obtained a contradiction. Thus, the theorem is proved.

An element $x \in L_{n}(B)$ does not belong to the image of $\phi_{1}$ if and only if its BrowderLivesay invariant $\partial(x)$ is non-trivial, where $\partial$ is the map in (4.2). An element $x \in L_{n}(B)$ does not belong to the image of $\phi_{2}$ if and only if either the first or the second BrowderLivesay invariant is non-trivial. The second Browder-Livesay invariant was introduced by Hambleton in [21. This invariant is defined only if the Browder-Livesay invariant is trivial. The iterated Browder-Livesay invariants were introduced by Kharshiladze (see [4, 9, 10]). The elements of $L_{n}(B)$ that do not belong to the image of $\phi_{j}$ for some $j$ (and only these elements) are detected by the iterated Browder-Livesay invariants, as immediately follows from [9, 10, 12. The fact that such elements cannot be realized by normal maps of closed manifolds was proved by Kharshiladze (see [9, 10]) by geometric methods. The proof of Theorem 4.4 actually uses the idea of the proof in 9 .

\section{REFERENCES}

[1] A. Bak and Yu.V. Muranov, Splitting along submanifolds, and L-spectra, Sovrem. Mat. Prilozh. No. 1, Topol. Anal. Smezh. Vopr. (Akad. Nauk Gruzii, Inst. Kibern. Tbilisi), 2003, 3-18; English transl. in J. Math. Sci. (N.Y.) 123 (2004), 4169-4184. MR2157601 (2006c:57029)

[2] I. Maleshich, Yu. V. Muranov, and D. Repovsh, Splitting obstruction groups in codimension 2, Mat. Zametki 69 (2001), 52-73; English transl. in Math. Notes 69 (2001), 46-64. MR1830982 (2002b:57039) 
[3] Yu. V. Muranov, Splitting obstruction groups and quadratic extensions of anti-structures, Izv. Ross. Akad. Nauk, Ser. Mat. 59, no. 6 (1995), 107-132; English transl. in Izv. Math. 59 (1995), 1207-1232. MR $1481617(98 \mathrm{i}: 57064)$

[4] Yu. V. Muranov, Splitting problem, Trudy Mat. Inst. Steklova 212 (1996), 123-146; English transl. in Proc. Steklov Inst. Math. 212 (1996), 115-137. MR1635031 (99e:57047)

[5] Yu. V. Muranov and A. F. Kharshiladze, Browder-Livesay groups of Abelian 2-groups, Mat. Sb. 181 (1990), 1061-1098; English transl. in Math. USSR Sb. 70 (1991), 499-540. MR.1076143 (91h:57023)

[6] Yu. V. Muranov and D. Repovsh, Groups of obstructions to surgery and splittings for a manifold pair, Mat. Sb. 188, no. 3 (1997), 127-142; English transl. in Sb. Math. 188 (1997), 449-463. MR:1462026 (98h:57059)

[7] Yu. V. Muranov and D. Repovsh, The groups LS and morphisms of quadratic extensions, Mat. Zametki 70 (2001), 419-424; English transl. in Math. Notes 70 (2001), 378-383. MR.1882251 (2002k:57082)

[8] Yu. V. Muranov, D. Repovsh, and F. Spaggiari, Surgery on triples of manifolds, Mat. Sb. 194, no. 8 (2003), 139-160; English transl. in Sb. Math. 194 (2003), 1251-1271. MR2034535 (2004m:57066)

[9] A. F. Kharshiladze, Iterated Browder-Livesay invariants and the oozing problem, Mat. Zametki 41 (1987), 557-563; English transl. in Math. Notes 41 (1987), 312-315. MR0897701 (88m:57043)

[10] A.F. Kharshiladze, Surgery on manifolds with finite fundamental groups, Uspekhi Mat. Nauk 42, no. 4 (1987), 55-85; English transl. in Russian Math. Surveys 42, no. 4 (1987), 65-103. MR0912061 $(88 \mathrm{j}: 57030)$

[11] I. Hambleton and Yu. V. Muranov, Projective splitting obstruction groups for one-sided submanifolds, Mat. Sb. 190, no. 10 (1999), 65-86; English transl. in Sb. Math. 190 (1999), 1465-1485. MR.1740157 (2000m:57048)

[12] I. Hambleton and A.F. Kharshiladze, A spectral sequence in surgery theory, Mat. Sb. 183, no. 9 (1992), 3-14; English transl. in Russ. Acad. Sci. Sb. Math. 77 (1994), 1-9. MR.1198831(93m:57036)

[13] W. Browder and G. R. Livesay, Fixed point free involutions on homotopy spheres, Bull. Amer. Math. Soc. 73 (1967), 242-245. MR0206965 (34:6781)

[14] W. Browder and F. Quinn, A surgery theory for G-manifolds and stratified sets, Proc. Int. Conf. Manifolds and Related Topics in Topology (Tokyo, 1973), Univ. of Tokyo Press, Tokyo, 1975, 27-36. MR0375348(51:11543)

[15] S. E. Cappell and J.L. Shaneson, A counterexample on the oozing problem for closed manifolds, Proc. Symp. Algebraic Topology (Aarhus 1978), Lecture Notes Math. vol. 763, Springer-Verlag, Berlin, 1979, 627-634. MR0561242 (81k:57033)

[16] S. E. Cappell and J. L. Shaneson, Pseudo-free actions. I, Proc. Symp. Algebraic Topology (Aarhus, 1978), Lecture Notes Math. vol. 763, Springer-Verlag, Berlin, 1979, 395-447. MR0561231 (81d:57034)

[17] A. Cavicchioli, Yu. V. Muranov, and D. Repovš, Spectral sequences in K-theory for a twisted quadratic extension, Yokohama Math. J. 46 (1998), 1-13. MR1670761 (99k:19003)

[18] A. Cavicchioli, Yu. V. Muranov, and D. Repovš, Algebraic properties of decorated splitting obstruction groups, Boll. Un. Mat. Ital. 4-B (2001), 647-675. MR1859427|(2002d:57027)

[19] M. M. Cohen, A course in simple-homotopy theory, Springer-Verlag, New-York, 1973. MR0362320 $(50: 14762)$

[20] S. C. Ferry, A. Ranicki, and J. Rosenberg (Eds.), Novikov conjectures, index theorems and rigidity, vols. 1, 2, London Math. Soc. Lecture Notes vols. 226, 227, Cambridge Univ. Press, Cambridge, 1995. MR 1388294 (96m:57002) MR 1388306 (96m:57003)

[21] I. Hambleton, Projective surgery obstructions on closed manifolds, Proc. Conf. Algebraic $K$-theory, Part II (Oberwolfach, 1980), Lecture Notes Math. vol. 967, Springer-Verlag, Berlin, 1982, 101-131. MR0689390 (84g:57026)

[22] I. Hambleton, A. Ranicki, and L. Taylor, Round L-theory, J. Pure Appl. Algebra 47 (1987), 131-154. MR0906966 (88i:18010)

[23] I. Hambleton, R. J. Milgram, L. Taylor, and B. Williams, Surgery with finite fundamental group, Proc. London Math. Soc. 56 (1988), 349-379. MR0922660 (89c:57043)

[24] I. Hambleton and E. K. Pedersen, Topological equivalence of linear representations for cyclic groups, Preprint Max-Planck Inst., Bonn, 1997; part I, Ann. Math. 161 (2005), 61-104; part II, Forum Math. 17 (2005), 959-1010. MR2195715 (2006h:19005b)

[25] W. Lück and A. Ranicki, Surgery transfer, Proc. Conf. Algebraic Topology and Transformation Groups (Göttingen, 1987), Lecture Notes Math. vol. 1361, Springer-Verlag, Berlin, 1988, 167-246. MR0979509 (90h:57041)

[26] W. Lück and A. Ranicki, Surgery obstructions of fibre bundles, J. Pure Appl. Algebra 81 (1992), 139-189. MR:1176019 (93h:19006) 
[27] Yu. V. Muranov and R. Jimenez, Homotopy triangulations of a manifold triple, Morphismos, Preprint Mexican Politech. Univ. 2006

[28] Yu. V. Muranov and R. Jimenez, Transfer map for triples of manifolds, Mat. Zametki, to appear; English transl. in Math. Notes.

[29] A. Ranicki, The total surgery obstruction, Proc. Symp. Algebraic Topology (Aarhus, 1978), Lecture Notes Math. vol. 763, Springer-Verlag, Berlin, 1979, 275-316. MR0561227 (81e:57034)

[30] A. Ranicki, Exact sequences in the algebraic theory of surgery, Math. Notes vol. 26, Princeton Univ. Press, Princeton, NJ, 1981. MR 0620795 (82h:57027)

[31] A. Ranicki, The L-theory of twisted quadratic extensions, Canad. J. Math. 39 (1987), 345-364. MR0899842 (89h:57027)

[32] A. A. Ranicki, Algebraic L-theory and topological manifolds, Cambridge Tracts in Math. vol. 102, Cambridge Univ. Press, Cambridge, 1992. MR.1211640 (94i:57051)

[33] R. Switzer, Algebraic topology - homotopy and homology, Grund. Math. Wiss. vol. 212, SpringerVerlag, Berlin, 1975. MR0385836 (52:6695)

[34] C. T. C. Wall, Surgery on compact manifolds, Academic Press, London, 1970; 2nd ed., Math. Surveys and Monographs, vol. 69, Amer. Math. Soc., Providence, RI, 1999. MR.1687388 (2000a:57089)

[35] S. Weinberger, The topological classification of stratified spaces, Univ. of Chicago Press, Chicago, 1994. MR1308714 (96b:57024)

Vitebsk State University, Vitebsk, Belarus'

E-mail address: ymuranov@mail.ru

Institute for Mathematics, Physics, and Mechanics, University of Ljubljana, Slovenia

E-mail address: dusan.repovs@uni-lj.si

Instituto de Matematicas, National Autonomous University of Mexico (UnAm), Morelos, MEXICO

E-mail address: rolando@aluxe.matcuer.unam.mx

Translated by E. KHUKHRO 